\title{
Apprentissage par projets à l'INSA ROUEN-NORMANDIE : Etude, réalisation et simulation d'amplificateurs « vintage » à lampes
}

\author{
Richard GRISEL, INSA ROUEN NORMANDIE, Département STPI, 685 Avenue de l'université \\ BP 0876801 Saint-Etienne-du-Rouvray Cedex \\ richard.grisel@insa-rouen.fr
}

Résumé : Le département Sciences et Techniques pour l'Ingénieur (STPI) de l'INSA ROUEN NORMANDIE permet à ses étudiants de deuxième année du cycle ingénieur $(\mathrm{Bac}+2)$ de préparer pendant 14 séances un projet par groupes de 6 ou 7 étudiants à raison d'une heure et demi encadrée par semaine. Cette séquence pédagogique permet aux étudiants de découvrir une première approche de travail par groupes. Depuis 2015 deux de ces projets concernent l'étude, la réalisation, la simulation et la vérification d'amplificateurs à lampes choisis comme étant des reproductions d'amplificateurs à lampes mythiques des années 1960. Le but de cette présentation est d'expliquer la démarche effectuée et de présenter l'ensemble des résultats obtenus.

\section{Introduction}

La séquence pédagogique se déroule en plusieurs phases. Les deux premières séances sont consacrées à une présentation synthétique des amplificateurs et des différents éléments constitutifs (préamplificateurs, amplificateurs, inverseurs, transformateurs, étages de sortie). Les étudiants se répartissent ensuite par groupe de 2 pour effectuer en parallèle les différentes activités nécessaires à la réalisation complète du projet. Ces activités peuvent être classées en 3 groupes :

- Etude théorique et simulation

- Montage et réalisation (électronique, câblage, montage)

- Mesures pratiques et comparaisons avec l'étude et la simulation.

A la fin du projet, certains étudiants font des essais avec leurs instruments (guitare et basse), il a aussi été fait appel à des musiciens disposant de guitares anciennes pour tester les amplificateurs [12]

Il est indiqué aux étudiants que les tensions d'alimentation sont très élevées et potentiellement dangereuses (plus de $250 \mathrm{VDC}$ ) et donc que toute manipulation devra se faire alimentation éteinte et en ayant vérifié que les condensateurs sont déchargés.

\section{Choix des amplificateurs}

Le choix initial des amplificateurs a été réalisé par l'enseignant à la suite d'un travail préparatoire devant permettre de répondre à plusieurs critères :

- caractéristique « historique » de l'amplificateur

- possibilité de se procurer un kit de montage pour la partie électronique avec tous les composants à un prix raisonnable et si possible dans une version faible puissance (inférieure à 15 Watts)

- documentation suffisante pour permettre un travail en autonomie avec un encadrement technique - une particularité différente au niveau technique pour chacun des amplificateurs. 
Ce travail préparatoire a permis de sélectionner 2 amplificateurs, le Fender Champ 5F1 et le VOX AC-30 :

- Le Fender Champ 5F1 a été choisi [1] car depuis sa première année de fabrication en 1948 il a accompagné des musiciens célèbres (Éric Clapton notamment pour des enregistrements en studio dans les années 1970 de titres tels que «Layla » et «I Shot the Sheriff », ZZ top et les Rolling Stones pour ne citer que les plus connus), il est disponible en kit, la documentation est plus que suffisante (notamment les schémas originaux des années 1950) et en termes de caractéristique techniques c'est un amplificateur de type «single-ended» en sortie avec une structure très simple mis à part la contre-réaction.

- Le VOX-AC30 a été choisi pour les mêmes raisons historiques [2] il est apparu un peu plus tard que le Fender (1958) mais a été largement utilisé lui aussi par des musiciens célèbres (The Shadows, John Lennon, Les Rolling Stones, Rory Gallagher, etc..) et a comme particularité de disposer d'un amplificateur différentiel suivi d'un étage de sortie de type push-pull.

\section{Présentation des caractéristiques du Fender Champ Tweed et approche pédagogique}

Le schéma original que l'on trouve pour le Fender Champ (avec les valeurs de polarisation) est le suivant :
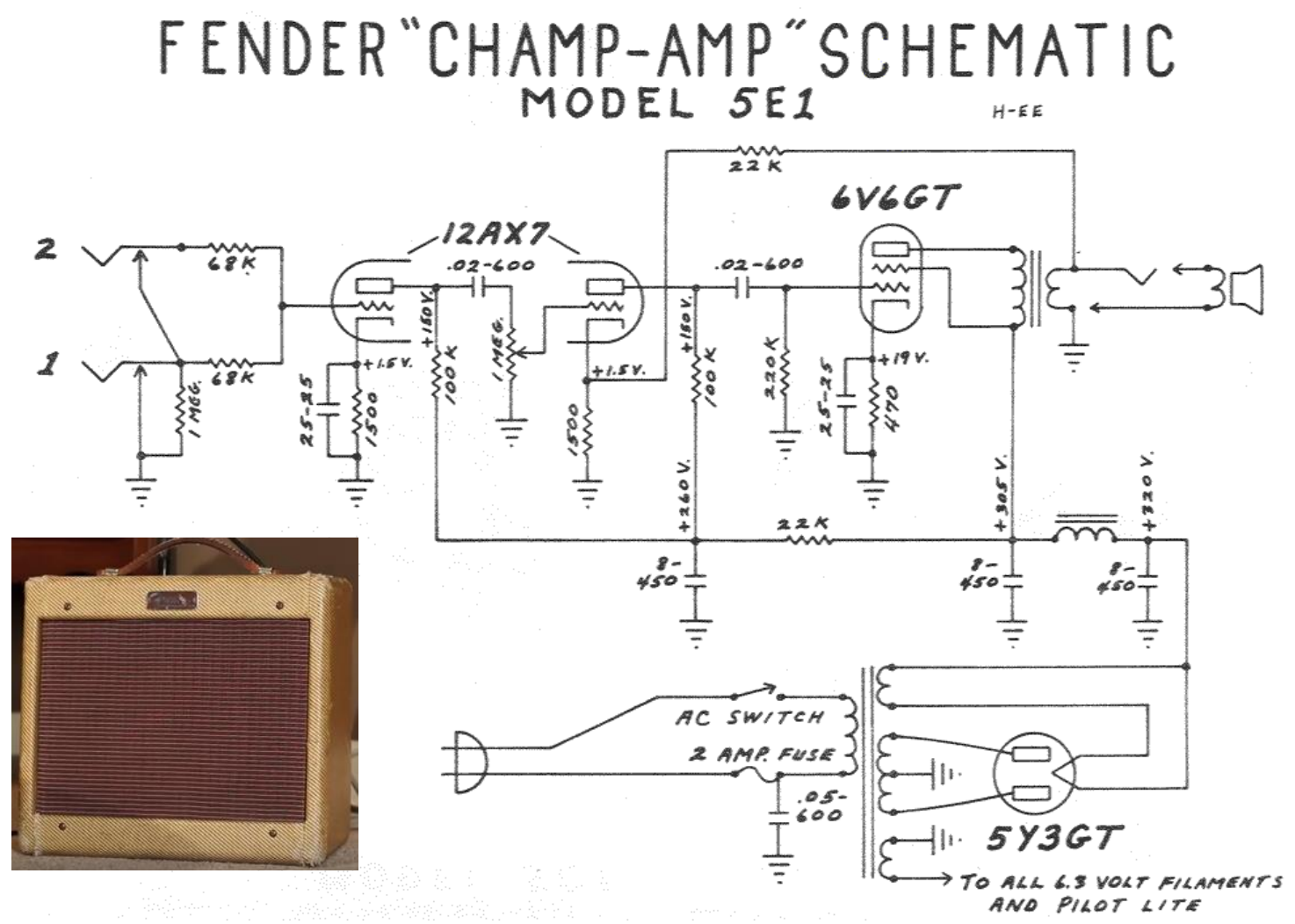

Figure 1 : Schéma original et photographie du Fender Champ amplificateur de 5 Watts

Le schéma correspondant à la réalisation est donné sur la Figure 2, ce schéma permet de faire avec les étudiants une présentation relativement simple des différents éléments constitutifs d'un amplificateur à lampes. 


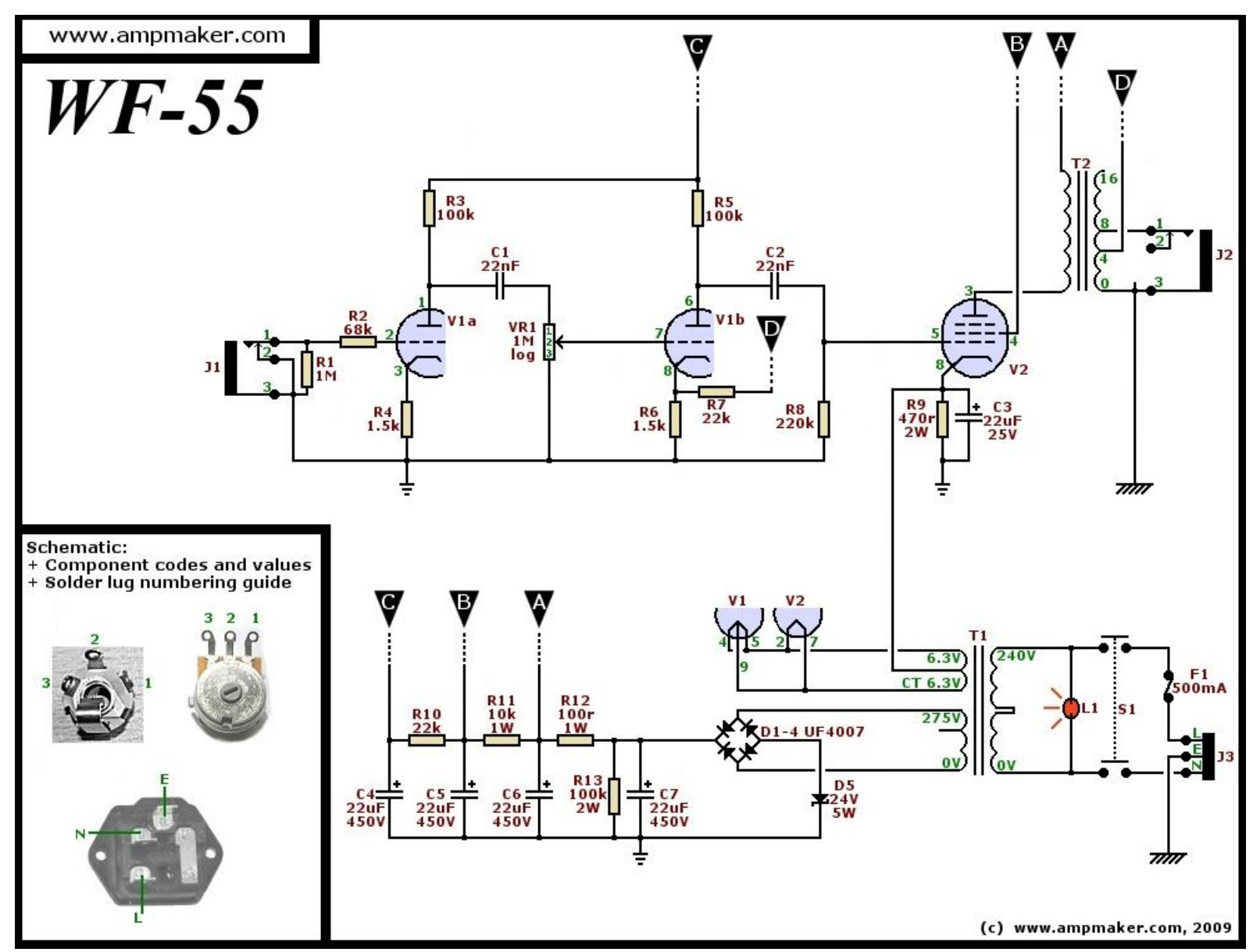

Figure 2 : Schéma de l'amplificateur modèle Champ Fender correspondant à la réalisation pour le projet

On peut remarquer qu'il n'y a que deux différences entre le schéma original de 1948 (Figure 1) et le schéma du prototype (Figure 2) :

La partie alimentation est réalisée avec un redresseur à diodes plutôt qu'avec un redresseur à lampes dans la version originale et il n'y a qu'une seule entrée haute impédance plutôt que deux entrées dans la version originale. La structure relativement simple de cet amplificateur permet de présenter les différentes parties du montage comme indiqué sur la Figure 3. 


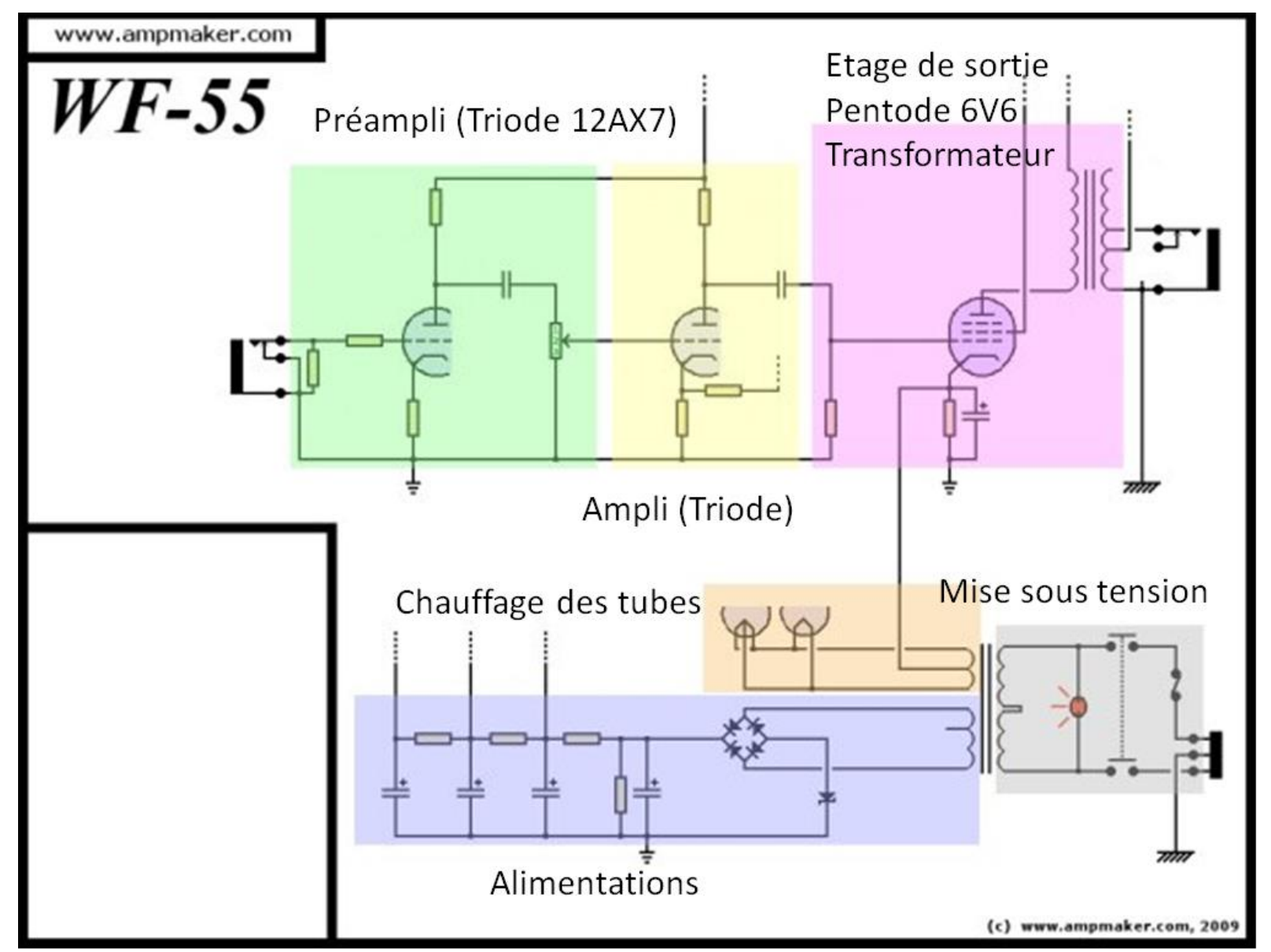

Figure 3 : Panneau de présentation aux étudiants des différents éléments du Fender

La présentation générale permet ensuite d'aborder les différents éléments.

\subsection{Approche de présentation du préamplificateur à triode $12 \mathrm{AX} 7$.}

La présentation de la fonction d'une triode se fait dans un premier temps de manière graphique à partir des courbes caractéristiques d'une triode [3] et en n'utilisant que des notions qu'ils sont censés connaître, notamment le cours d'électricité de première année dispensé au semestre S1 sur 14 semaines (12h de cours, $21 \mathrm{~h}$ de TD, 18h de TP). Le schéma de la Figure 4 est donc présenté avec les différents courants et tensions : 


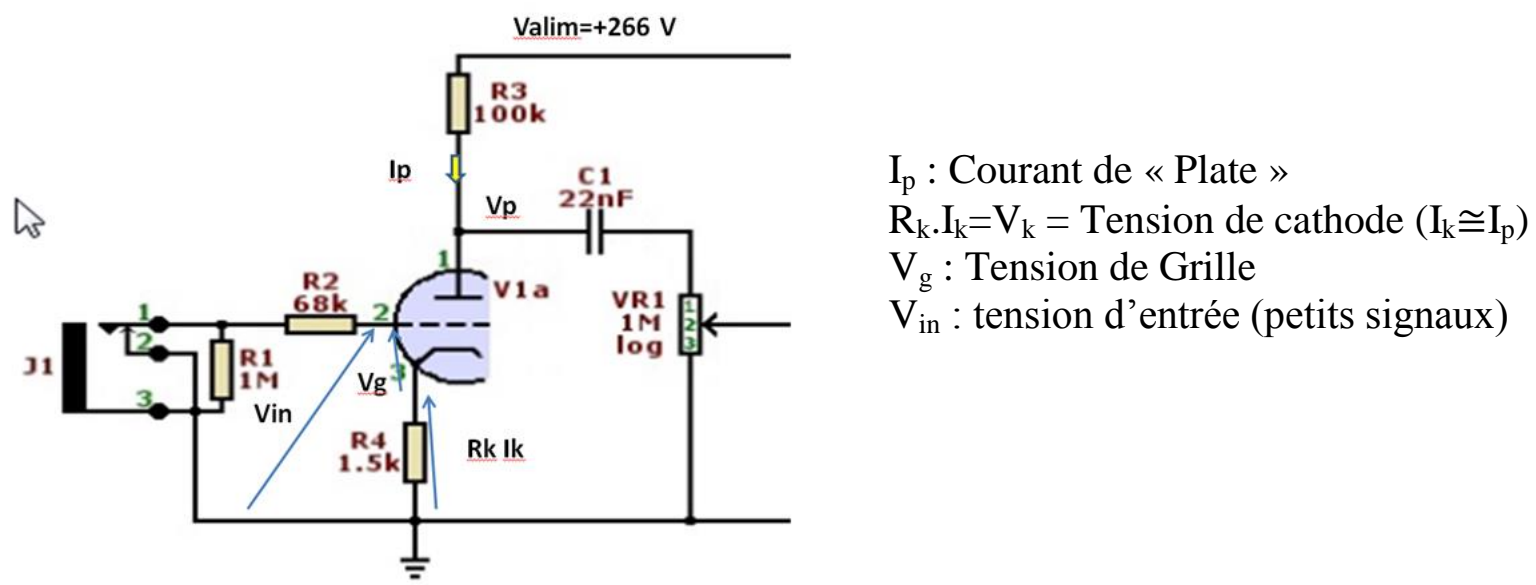

Figure 4 : Schéma du Préampli avec les notations utilisées

Le réseau caractéristique d'une triode (Figure 5) donne le courant $\mathrm{I}_{\mathrm{p}}$ («Plate current») en fonction de la tension $V_{p}$ (Plate voltage) pour différentes tensions de grille $\left(V_{g}\right)$. On a comme hypothèse $I_{k} \cong I_{p}$ (pas de courant dans la grille). L'analyse se fait en deux temps :

\section{- Analyse du point de polarisation du montage :}

Les condensateurs sont des interrupteurs ouverts, la tension $V_{\text {in }}$ est nulle, on va donc avoir la tension de grille égale à $0-\left(\mathrm{R}_{\mathrm{k}} \cdot \mathrm{I}_{\mathrm{k}}\right)$ donc une tension négative.

On a l'équation de la droite dite « de charge » :

$$
\mathrm{V}_{\text {alim }}-\mathrm{V}_{\mathrm{p}}=\text { R3. } \mathrm{I}_{\mathrm{p}} \text { soit } \mathrm{I}_{\mathrm{p}}=\frac{\mathrm{V}_{\text {alim }}-\mathrm{V}_{\mathrm{p}}}{R 3}=\frac{266-\mathrm{V}_{\mathrm{p}}}{100 k \Omega}
$$

On trace cette droite de charge (en rouge) (polarisation à $266 \mathrm{~V}$ ) avec deux points : 1 point a 0 $266 / 100 \mathrm{k}=2,66 \mathrm{~mA}$ et 1 point à $(266 \mathrm{~V}, 0 \mathrm{~mA})$.

La deuxième droite de charge (en bleu) correspond à l'équation

$\mathrm{I}_{\mathrm{k}}=\frac{\mathrm{V}_{\mathrm{g}}}{500} \quad\left(\mathrm{R}_{\mathrm{k}}=500 \Omega\right)$

On trace deux points pour l'un pour $\mathrm{V}_{\mathrm{G}}=-1 \mathrm{~V}$ et l'autre pour $\mathrm{V}_{\mathrm{G}}=-2 \mathrm{~V}$. L'intersection donne le point de polarisation à environ $\mathrm{V}_{\mathrm{G}}=-1,4 \mathrm{~V}$ (ce qui sera mesuré plus tard sur la maquette) et donc un courant $\mathrm{I}_{\mathrm{p}}=1,4 \mathrm{~mA} / 1,5 \mathrm{k} \Omega=0,933 \mathrm{~mA}$. 


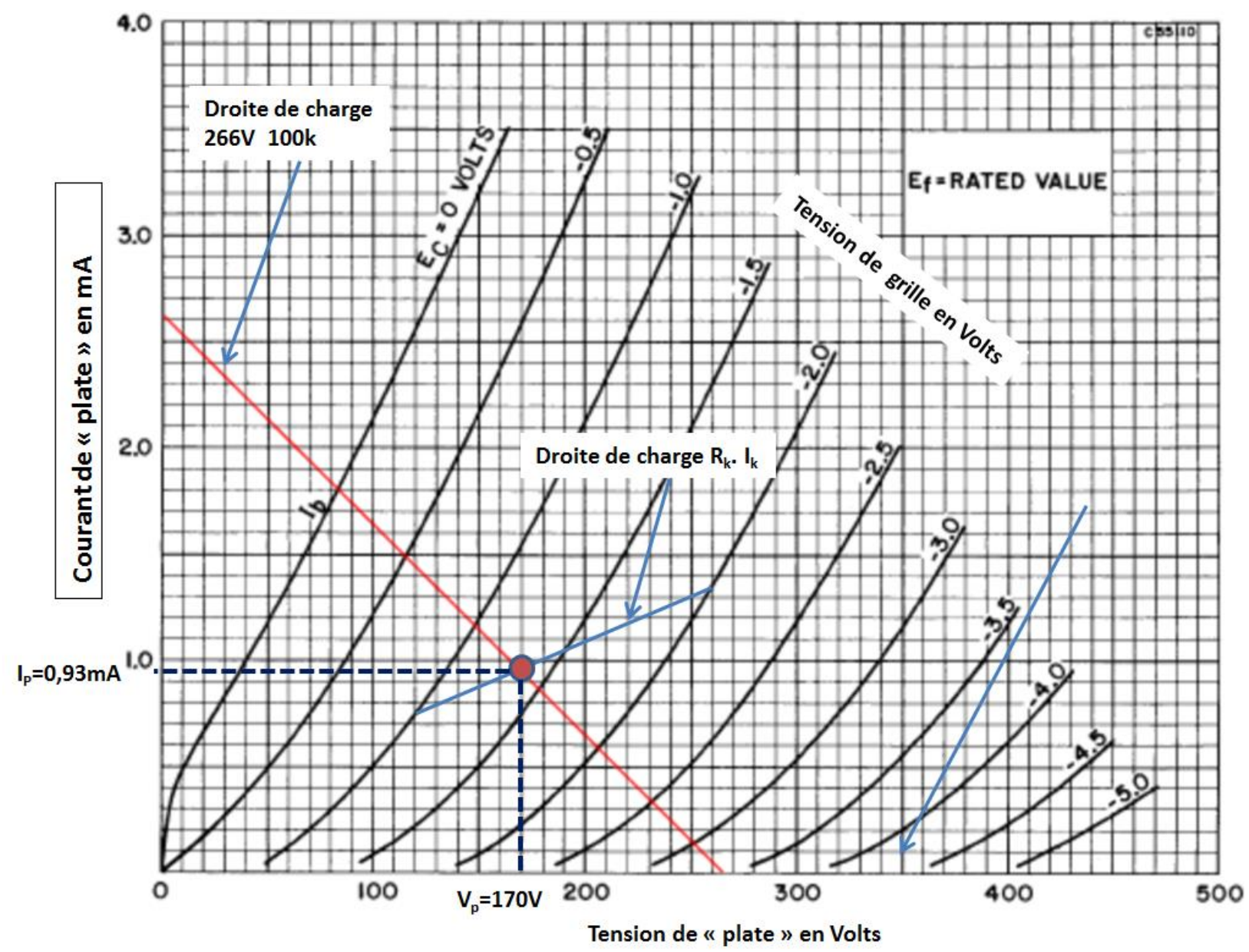

Figure 5 : Polarisation de la triode du préamplificateur

\section{- Calcul du gain du montage : caractéristiques dynamiques dites « petits signaux » alternatifs.}

Le schéma équivalent en mode alternatif petits signaux correspond à l'application d'une tension sinusoïdale de faible amplitude (quelques dizaines de $\mathrm{mV}$ ) sur $\mathrm{V}_{\mathrm{in}}$. L'apparition de cette tension va provoquer de faibles variations autour du point de fonctionnement. Par contre en alternatif petits signaux les condensateurs se comportent comme des courts-circuits et les alimentations continues se comportent elles aussi comme des courts-circuits. Le schéma équivalent devient donc le suivant (Figure 6) avec indication par $\Delta \mathrm{V}$ des petites variations autour de la tension de polarisation.

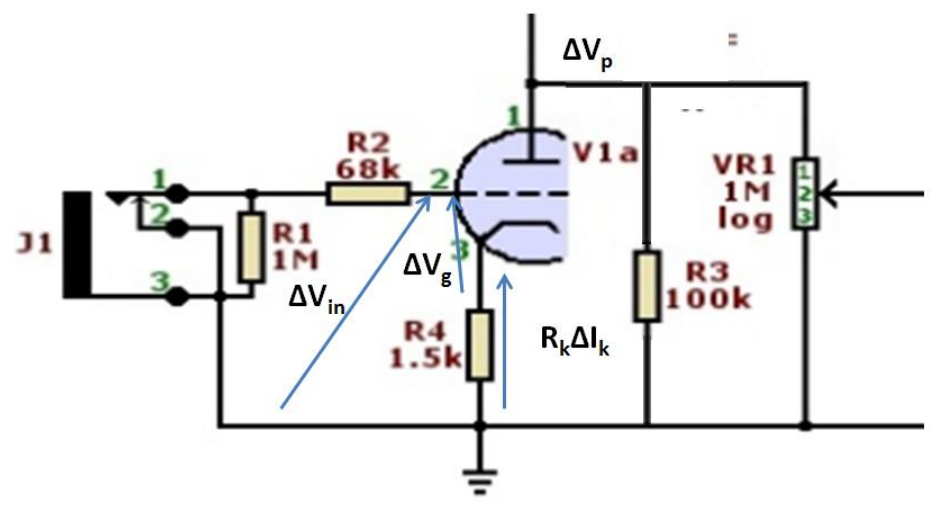

Figure 6 : Schéma équivalent petits signaux

On notera donc par la suite $\Delta \mathrm{V}$ les petites variations en tension autour du point de polarisation et $\Delta \mathrm{I}$ les petites variations en courant autour du point de polarisation. Il est ensuite précisé aux étudiants que ce schéma amène deux conséquences : 
Première conséquence : la droite de charge pour les petits signaux alternatifs n'est plus la droite de charge en continu, c'est la droite de charge qui correspond à une résistance de charge égale à R3 en parallèle sur la résistance du potentiomètre de volume de $1 \mathrm{M} \Omega$. Cette résistance de charge est donc de $100 \mathrm{k} \Omega / / 1 \mathrm{M} \Omega=90$ $900 \Omega$. On trace donc cette droite de charge sur la caractéristique de la triode (Figure 7) :

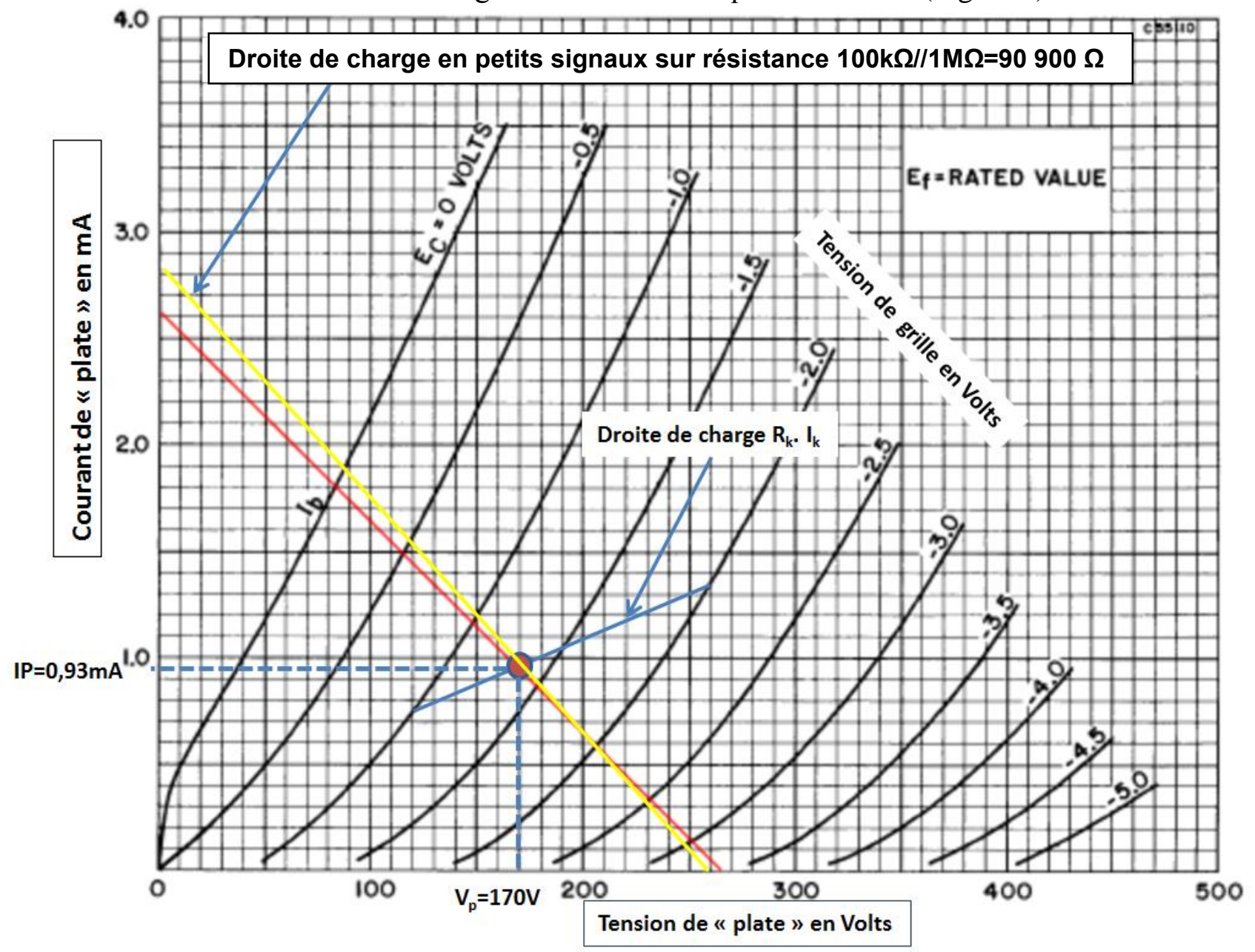

Figure 7 : Droite de charge dite petits signaux alternatifs

Deuxième conséquence : la variation de la tension $\Delta \mathrm{V}_{\text {in }} \mathrm{s}$ 'écrit :

$$
\Delta \mathrm{V}_{\text {in }}=\Delta \mathrm{V}_{\mathrm{g}}+\mathrm{R}_{\mathrm{k}} \cdot \Delta \mathrm{I}_{\mathrm{k}}=\Delta \mathrm{V}_{\mathrm{g}}+\mathrm{R}_{\mathrm{k}} \cdot \Delta \mathrm{I}_{\mathrm{p}}
$$

On trace donc sur la courbe de transfert le $\Delta \mathrm{I}_{\mathrm{k}}$ obtenu pour un $\Delta \mathrm{V}_{\mathrm{g}}$ de $1 \mathrm{~V}$, ce qui nous donne $\Delta \mathrm{I}_{\mathrm{k}}=0,7 \mathrm{~mA}$ (voir Figure 8) : 


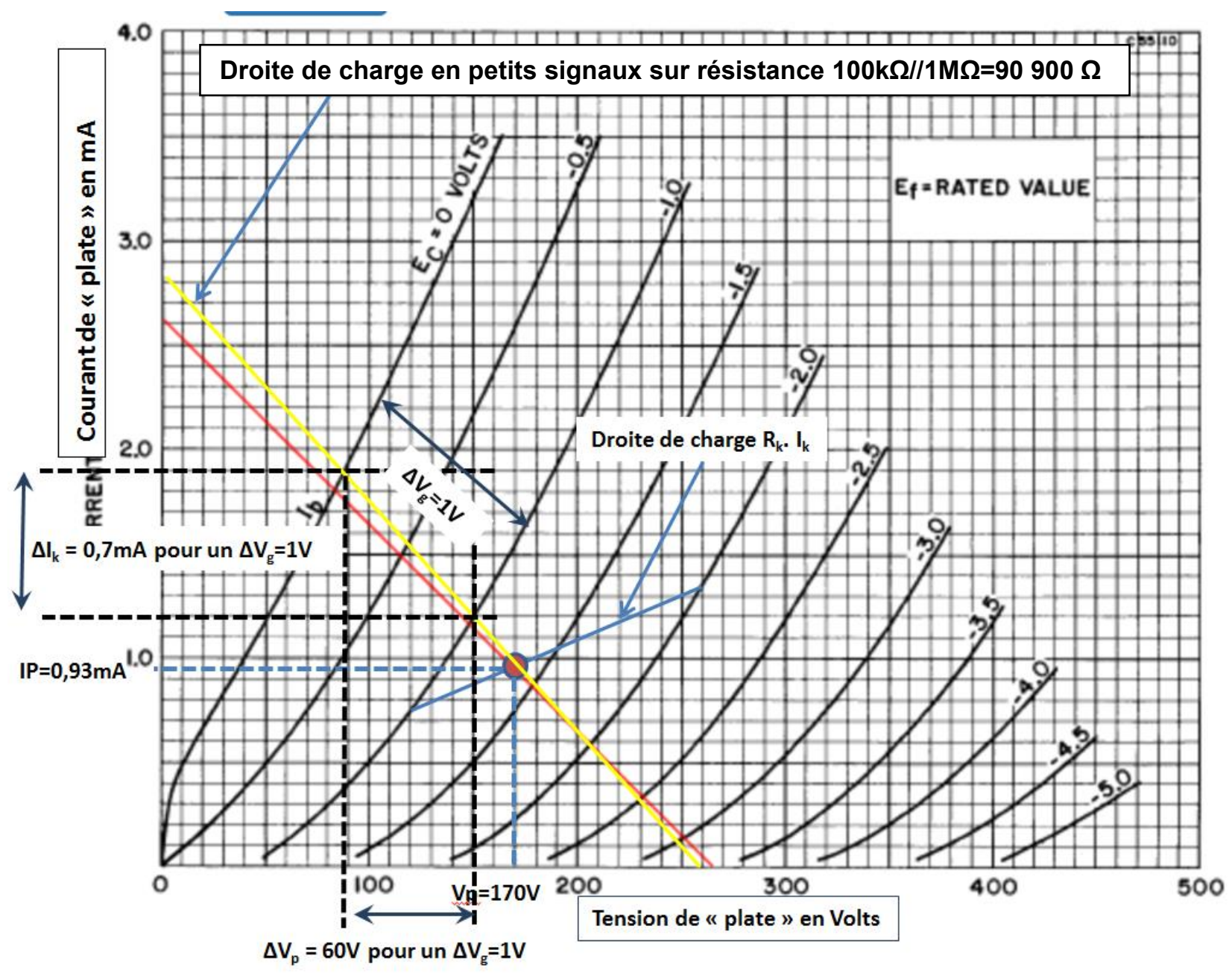

Figure 8 : Effet de la résistance de cathode

Cela correspond donc à :

$$
\Delta \mathrm{V}_{\mathrm{in}}=\Delta \mathrm{V}_{\mathrm{g}}+\mathrm{R}_{\mathrm{k}} \cdot \Delta \mathrm{I}_{\mathrm{k}}=1 \mathrm{~V}+0,7 \mathrm{~mA} \cdot 1,5 \mathrm{k} \Omega=2,05 \mathrm{~V}
$$

Sur cette même caractéristique on trace le $\Delta \mathrm{V}_{\mathrm{p}}$ obtenu pour ce $\Delta \mathrm{V}_{\mathrm{g}}$ de $1 \mathrm{~V}$ et donc pour un $\Delta \mathrm{V}_{\text {in }}$ de $2,05 \mathrm{~V}$, il est mesuré à $60 \mathrm{~V}$.

Le gain en tension de ce montage en module (les valeurs ne sont pas données en $\mathrm{dB}$ ) est donc de :

$$
\left|\frac{\Delta V p}{\Delta V i n}\right|=\text { gain en tension petits signaux }=\frac{60}{2,05}=29,27
$$

On parle de module car si l'on analyse ce qui se passe en regardant l'effet de la sinusoïde d'entrée on constate qu'une augmentation de la tension d'entrée se traduit par une diminution de la tension de sortie et inversement une diminution par une augmentation.

On écrit donc :

$$
\frac{\Delta V p}{\Delta V i n}=\text { gain en tension petits signaux }=\frac{-60}{2,05}=-29,27
$$

Les étudiants sont invités à aller vérifier cette valeur sur un calculateur en ligne :

https://www.ampbooks.com/mobile/amplifier-calculators/cathode-capacitor/

En mettant les valeurs $R_{G}=1 M \Omega$ (potentiomètre) $R_{L}=100 k \Omega=R_{3} R_{k}=1,5 k \Omega$ et $C_{x}$ très faible pour indiquer l'absence de condensateur en parallèle sur $R_{k}$ (impédance très élevée). Le gain donné en module est équivalent à celui trouvé graphiquement. 


\subsection{Approche de présentation de l'amplificateur à triode 12AX7.}

Le schéma de la Figure 9 permet d'introduire le deuxième étage d'amplification à la suite du préampli.

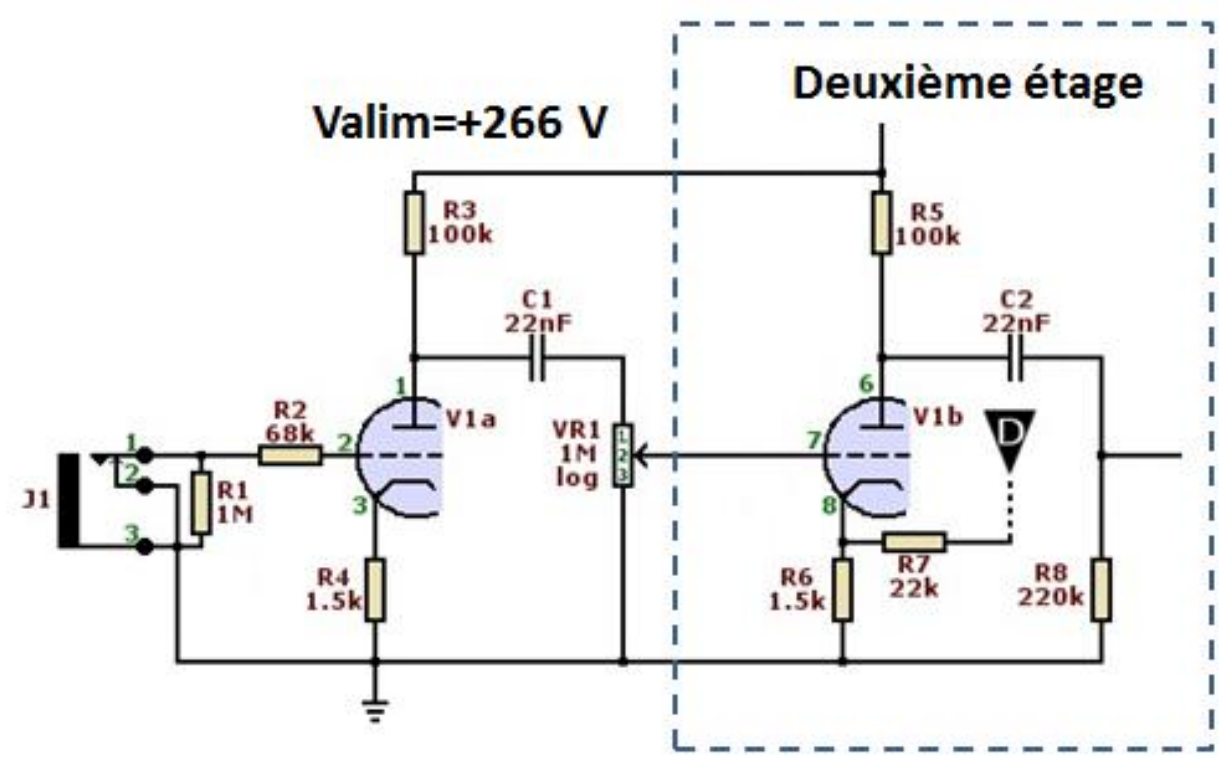

Figure 9 : Positionnement de l'Amplificateur (Deuxième étage)

Le deuxième étage présente la particularité d'avoir une contre réaction ( «feedback») via le pont résistif R7/R6, le point D étant pris en sortie du transformateur audio alimentant le haut-parleur (sortie 4 ohms). On commence donc par une étude sans cette contre-réaction qui est calée sur le modèle de l'étude du préamplificateur. Cela va donner un gain en boucle ouverte (B.O.)

On est donc dans une configuration équivalente au montage précédent mais avec une résistance de charge en mode «petits signaux » de $100 \mathrm{k} \Omega / / 220 \mathrm{k} \Omega$ soit $68,75 \mathrm{k} \Omega$.

Cela donne comme valeur

$$
\frac{V_{o}}{V_{\text {in }}}(\text { B. O. })=-24,314
$$

\section{Etude de la contre-réaction :}

Le schéma d'une contre réaction fait apparaître le gain en Boucle ouverte (GBO) et le gain de la chaîne de retour $\mathrm{H}$. Le gain dit en boucle fermée $(\mathrm{GBF})$ est égal à la formule :

$$
\mathbf{G B F}=\frac{\text { GBO }}{\mathbf{1}+\text { H. GBO }}
$$

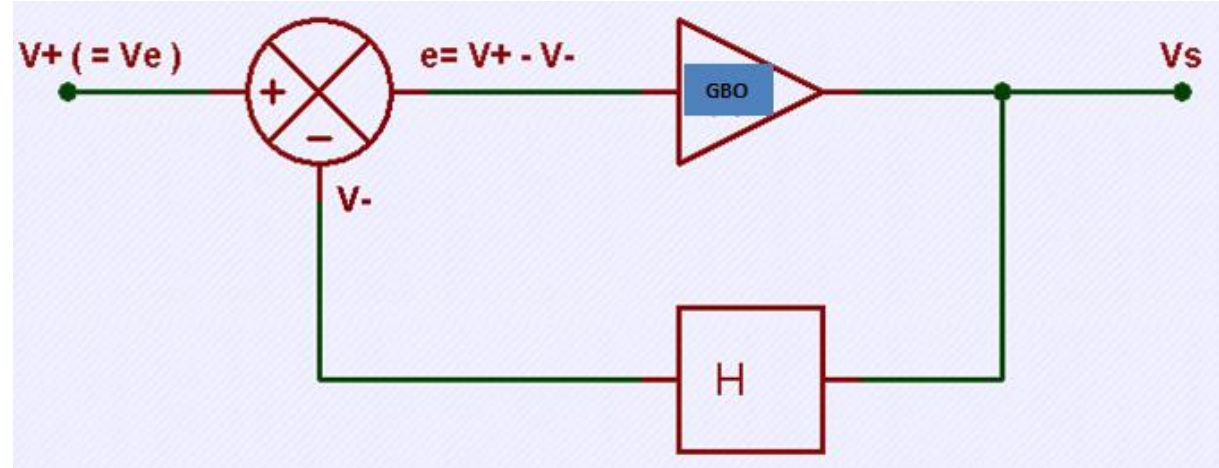


On explique aux étudiants que le gain de la chaîne de retour $(\mathrm{H})$ est égal au gain de l'étage de puissance (17) multiplié par le rapport de transformation du transformateur audio (1/35,35 ce rapport est inférieur à 1 car le transformateur abaisse la tension) multiplié par le rapport dû au diviseur de tension R7/R6 (et à la résistance d'entrée de la cathode) $(0,0307)$. On a donc :

$\mathrm{H}=17$. (1/35,35). 0,0307 = 0,014763. Soit H.GBO=0,3589

Et donc le gain en boucle fermé :

$$
\mathrm{GBF}=\frac{24,314}{1+0,3589}=17,89
$$

Le lien vers le site : https://www.ampbooks.com/mobile/classic-circuits/fender-champ-5e1-negativefeedback/ est conseillé aux étudiants qui veulent approfondir cette question (L'auteur de cet article ayant contribué à sa rédaction).

\subsection{Approche de présentation de l'étage de sortie (puissance).}

Le schéma de l'étage de sortie est présenté sur la Figure 10. La lampe utilisée est une pentode 6V6 [4] .

Il est expliqué aux étudiants que la présence du transformateur de sortie audio se caractérise pour les calculs par :

- Une résistance série d'environ $300 \Omega$ que l'on négligera dans les calculs approximatifs

- Une résistance dynamique de sortie (active seulement en mode petits signaux) qui fait partie des caractéristiques du transformateur utilisé. Pour le transformateur concerné cette résistance dynamique est de $5000 \Omega$ quelle que soit l'impédance de charge (puisqu'il y a une sortie 4 ohms et une sortie 8 ohms).

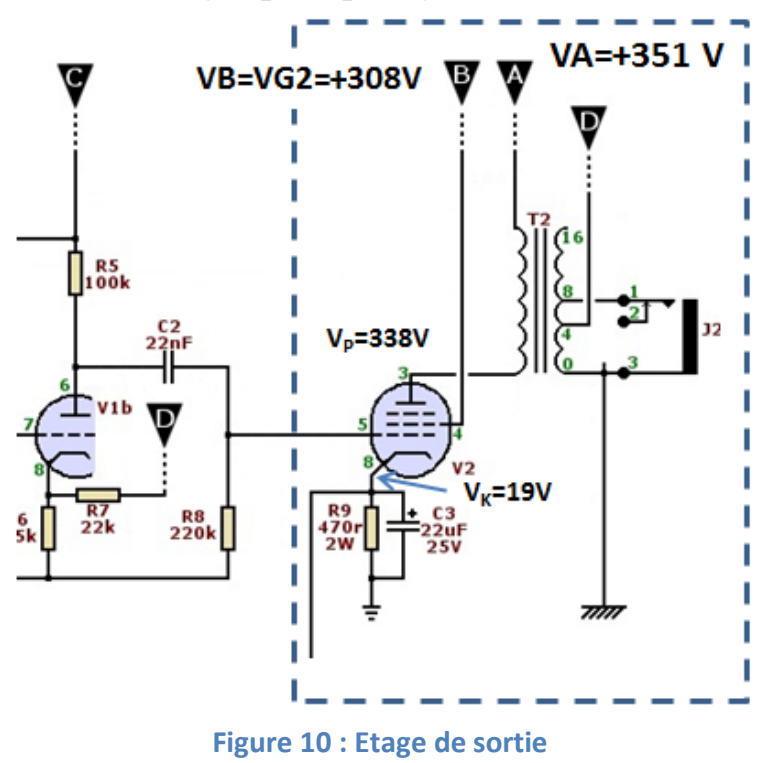

Les données de polarisation sont les suivantes :

$\mathrm{V}_{\mathrm{p}}$ (anode) $=338 \mathrm{~V}$, cette tension est imposée par l'alimentation de $351 \mathrm{~V}$ (VA) et il y a une chute de tension dans l'enroulement du primaire du transformateur.

$\mathrm{V}_{\mathrm{G} 2}=308 \mathrm{~V}$ vient de la sortie $\mathrm{B}$ de l'alimentation.

$\mathrm{V}_{\mathrm{K}}=19 \mathrm{~V}$.

La tension donnée dans les caractéristiques Fender pour une polarisation par la résistance de $470 \Omega$ (cathode) est de 19 volts (voir Figure 1), donc la loi d'Ohm donne un courant de 19/470=40 mA. Ce courant est la somme du courant de «plate» et du courant de «screen » (il n'y a pas de courant dans la grille numéro 1). On présente donc la courbe «plate-current» en fonction de «grid-number1 » sur laquelle la courbe à $\mathrm{V}_{\mathrm{G} 2}=300 \mathrm{~V}$ est estimée. Cela donne un courant de "plate" de $36 \mathrm{~mA}$ (Figure 11) 


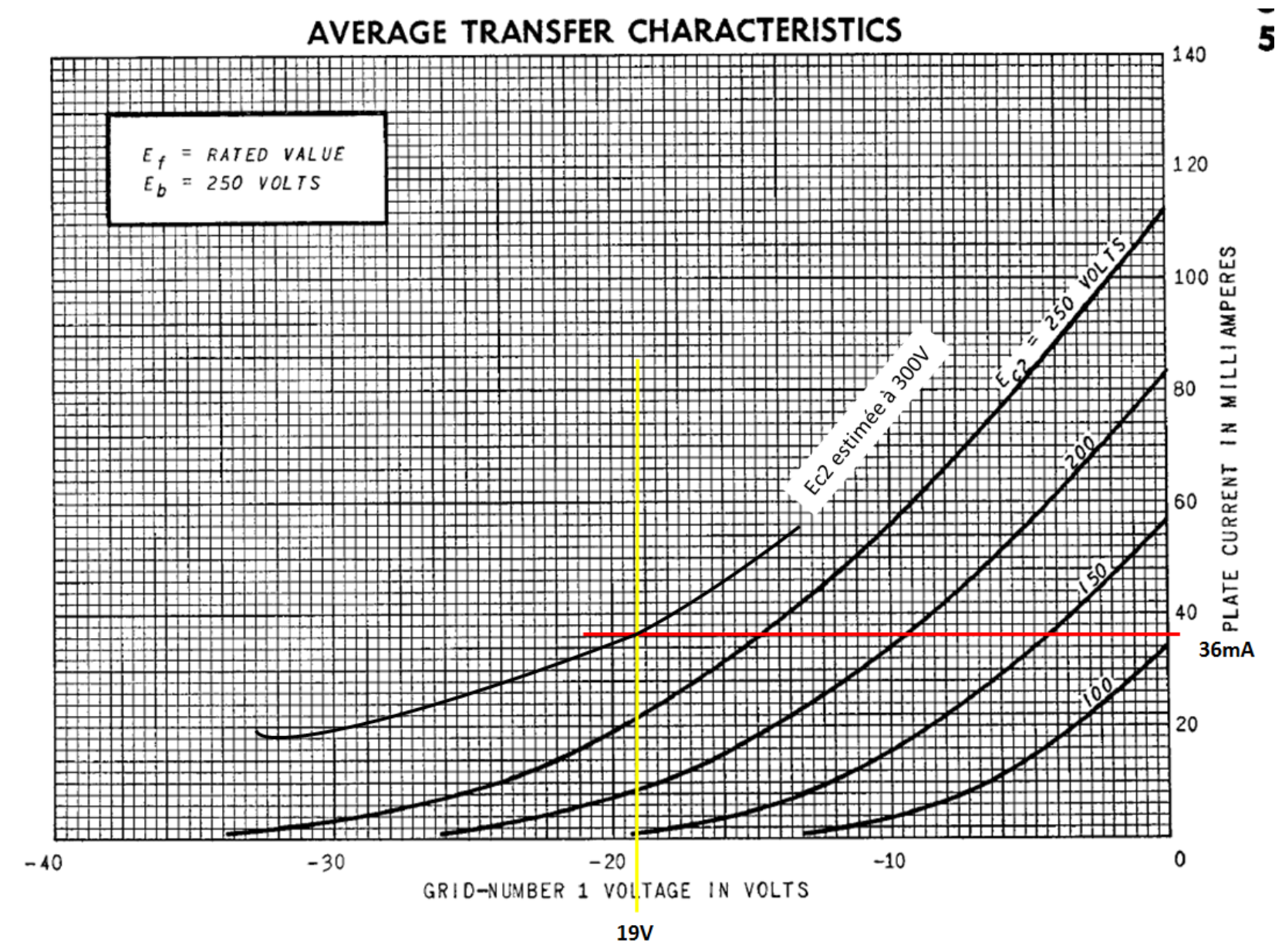

Figure 11 : Caractéristiques graphiques $6 \mathrm{~V} 6$ de sortie

Et donc on présente aussi la courbe « screen-current» fonction de $\mathrm{V}_{\mathrm{G} 1}$ à $\mathrm{V}_{\mathrm{p}}=$ constante ce qui permet de voir que l'on a bien $4 \mathrm{~mA}$ soit $36+4=40 \mathrm{~mA}$ (Figure 12). 


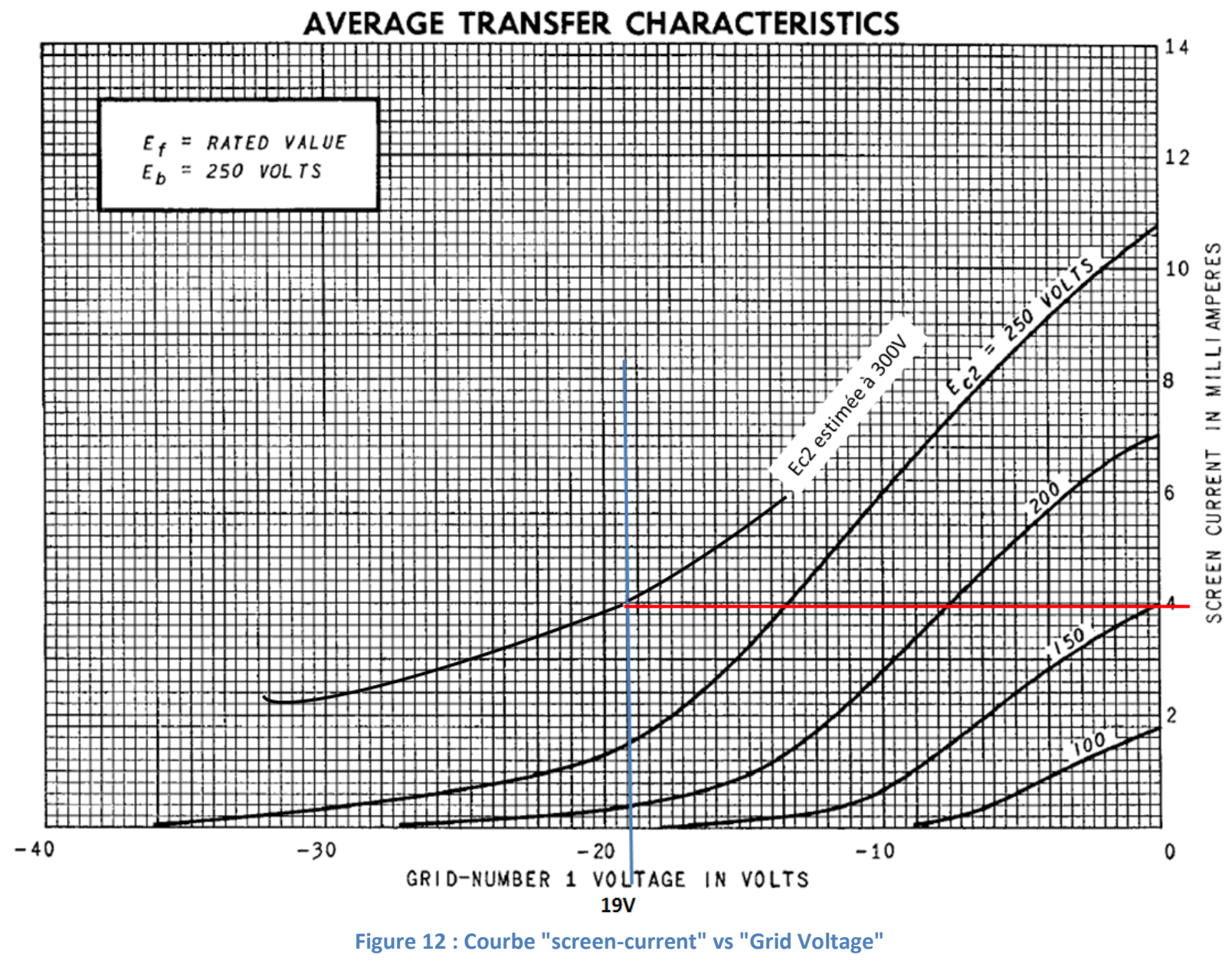

On a donc un point de polarisation à $36 \mathrm{~mA}$ pour $\mathrm{V}_{\mathrm{G}}=-19 \mathrm{~V}\left(\Delta \mathrm{V}_{\mathrm{in}}=0\right)$ pour $\mathrm{V}_{\mathrm{p}}=338 \mathrm{~V}$ (donné dans les caractéristiques) ce qui permet de placer les points sur la courbe de transfert avec toujours une estimation de la courbe pour $\mathrm{V}_{\mathrm{G} 2}=300 \mathrm{~V}$. Sur cette courbe on trace la droite de charge correspondant à la résistance du primaire en dynamique, soit $5 \mathrm{~K} \Omega$ ainsi que la puissance maximum censée être à $15 \mathrm{~W}$ pour la $6 \mathrm{~V} 6$ utilisée de manière à montrer que l'on fonctionne en dessous de la limite de puissance. 


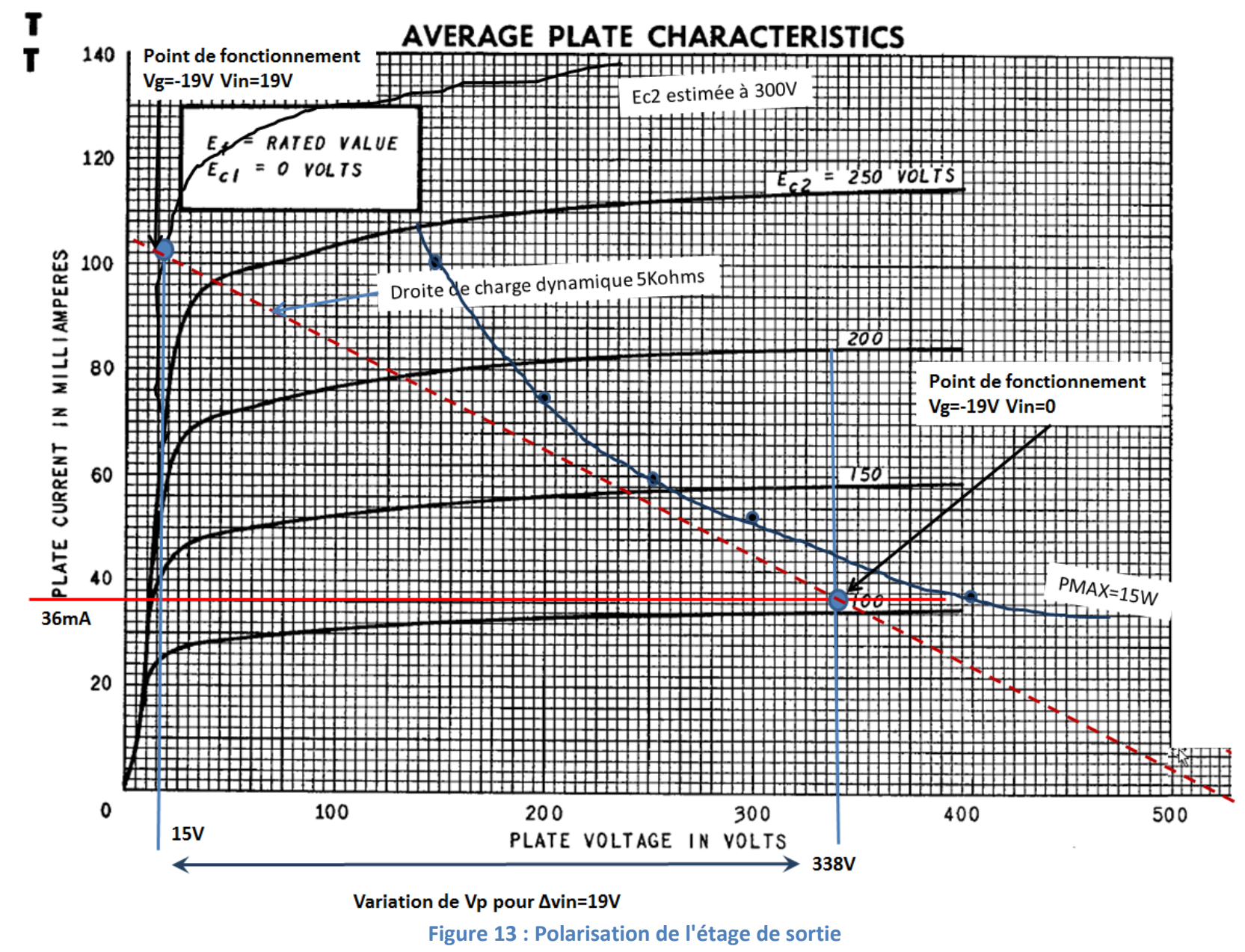

Le point de polarisation à $(36 \mathrm{~mA}, 338 \mathrm{~V})$ et le point de polarisation correspondant à l'excursion maximale que peut prendre $\mathrm{V}_{\text {in }}$ (soit $19 \mathrm{~V}$ ) qui se trouve suivant la droite de charge à $(102 \mathrm{~mA}, 15 \mathrm{~V})$ permettent de calculer le gain :

$$
\text { Gain } 6 \text { V6 }=\frac{338-15}{119}=17
$$

Le transformateur de sortie est un Hammond série 125 SE [5] qui donne une résistance dynamique au primaire de $5000 \Omega$ pour $8 \Omega$ ou $4 \Omega$ au secondaire (il y a plusieurs sorties, voir Figure 14).

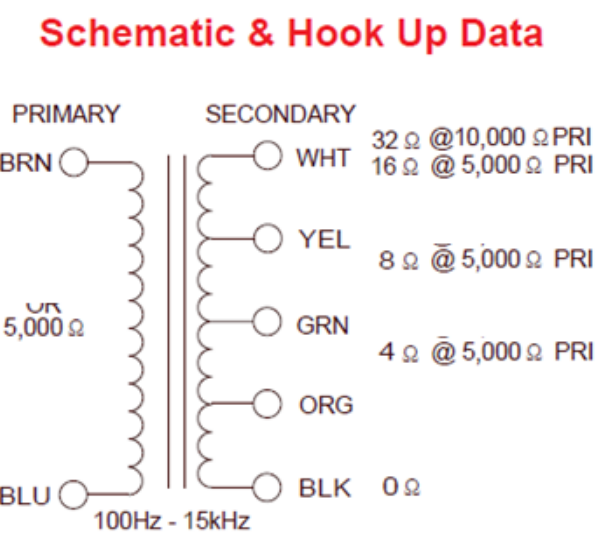

Figure 14 : Données du transformateur de sortie

Il est expliqué aux étudiants que le rapport de transformation entre le primaire et le secondaire s'exprime sous la forme de la racine carrée du rapport des impédances on écrit donc : 


$$
\frac{N 2}{N 1}=\sqrt{\frac{Z 2}{Z 1}}=\sqrt{\frac{8}{5000}}=\sqrt{\frac{1}{625}}=\frac{1}{25}(\text { pour } 8 \Omega) \quad \sqrt{\frac{1}{1250}}=\frac{1}{35,35} \operatorname{pour} 4 \Omega
$$

Le transformateur atténue l'amplitude mais augmente d'un facteur équivalent le courant de sortie assurant la puissance de sortie.

Conclusion : Pour $8 \Omega$ on a donc le rapport entre la sortie $8 \Omega$ et l'entrée qui a une valeur théorique de $30 * 17 * 17 / 25=347$ (soit $50 \mathrm{~dB})$. Pour $4 \Omega$ ce rapport est de $30 * 17 * 17 / 35,35=245,26(47,8 \mathrm{~dB})$

Remarque : Après la présentation théorique, les étudiants se divisent en groupes de 2 pour réaliser le montage de l'amplificateur, le câblage des alimentations et du boitier ainsi que la partie d'étude par la simulation. Ils sont supervisés par l'équipe technique de l'INSA et par le responsable de projet et travaillent en autonomie.

\subsection{Utilisation du simulateur pour la vérification des caractéristiques}

Un schéma de départ (le préamplificateur) est fourni aux étudiants (avec notamment les bibliothèques de composant pour les tubes) pour qu'ils puissent s'approprier le fonctionnement du simulateur choisi qui est LTSPICE (http://www.analog.com/en/design-center/design-tools-and-calculators.html ). Le modèle du transformateur de sortie leur est aussi fourni. Cela leur permet rapidement d'arriver au schéma de simulation de l'ensemble (Figure 15):

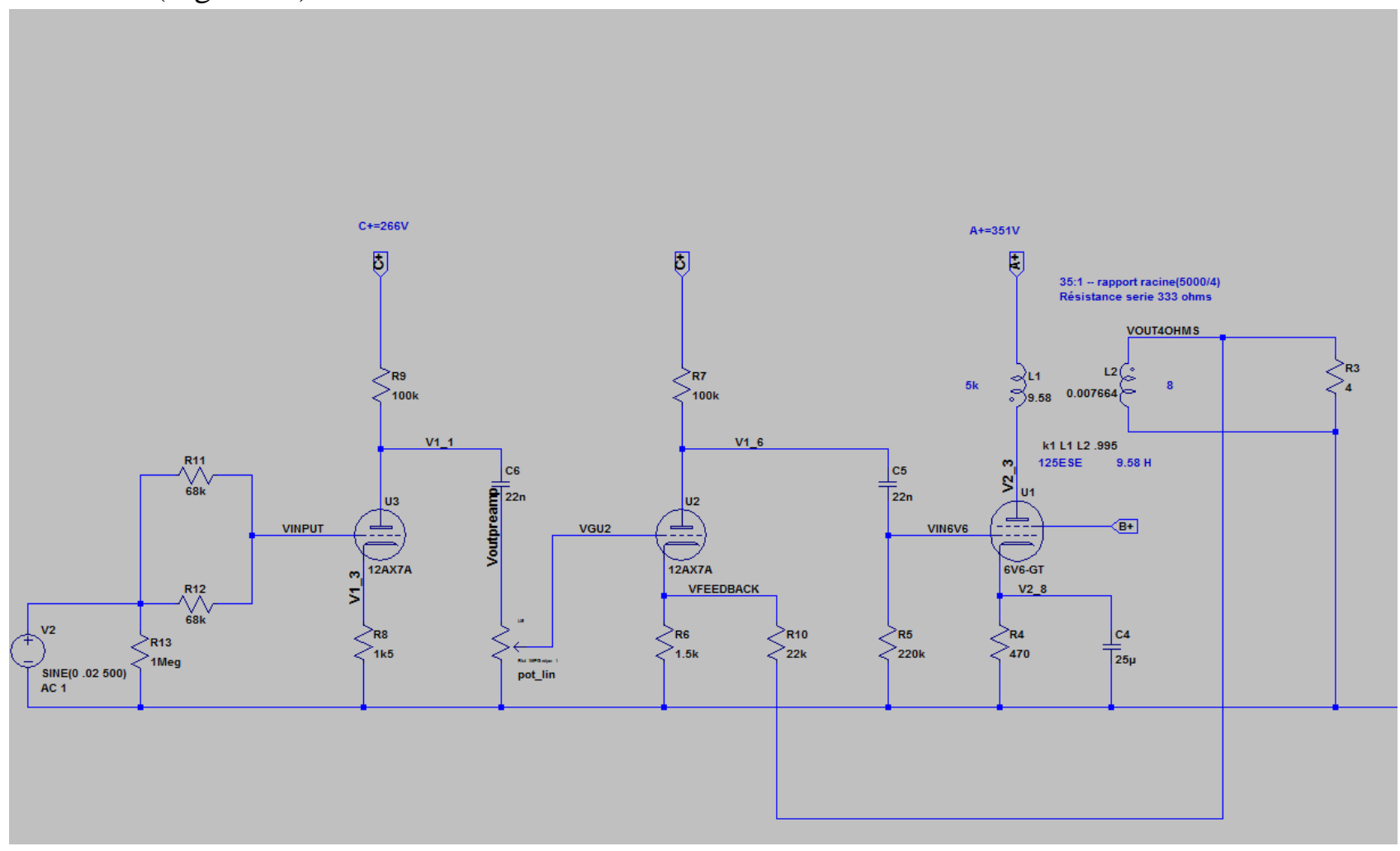

Figure 15 : Schéma LTSPICE réalisé par les étudiants

Cela leur permet de vérifier les valeurs de gain (pour une tension d'entrée de $40 \mathrm{mV}$ crête-crête à $500 \mathrm{~Hz}$ ) : 


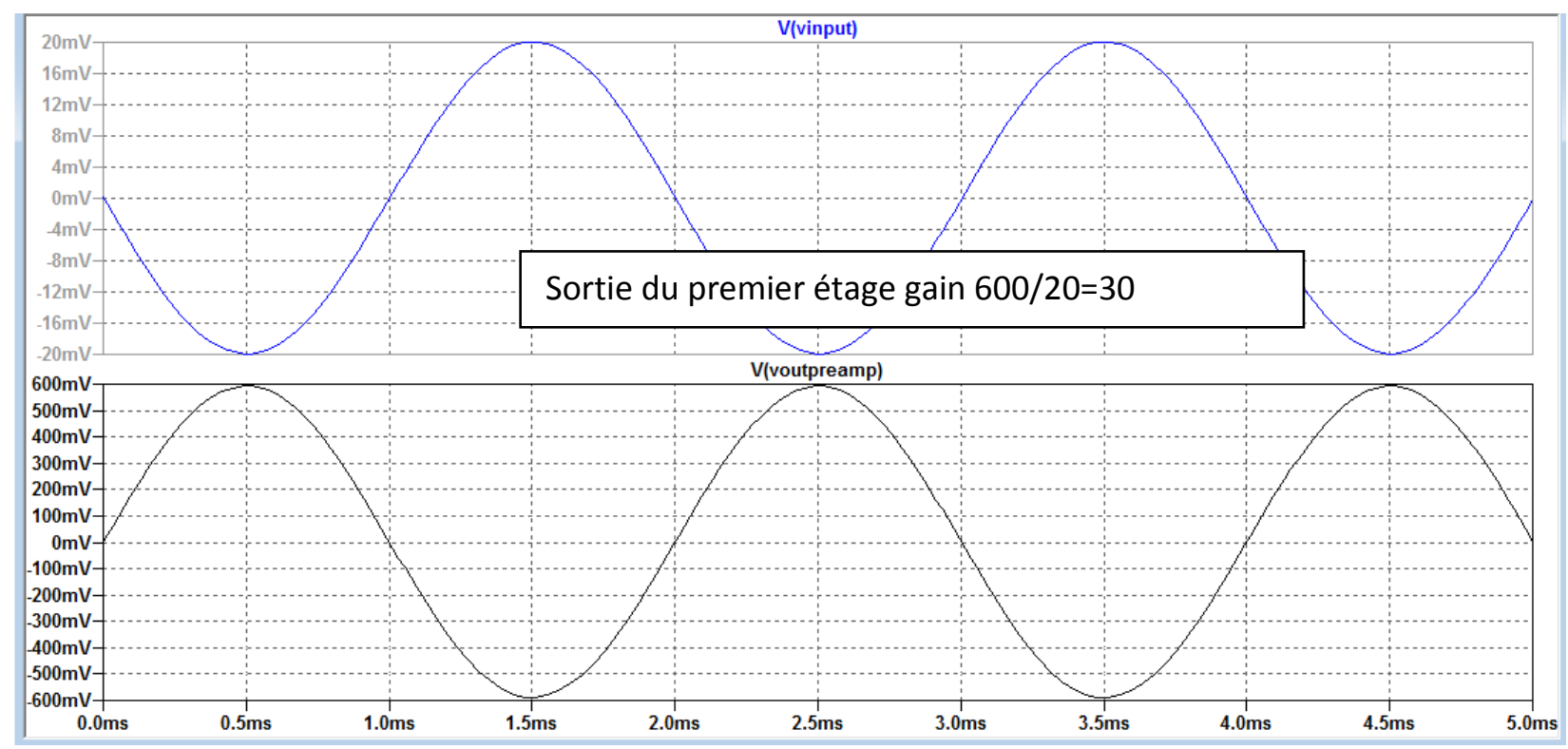

Figure 16 : Haut : Entrée du préampli Bas : Sortie du préampli

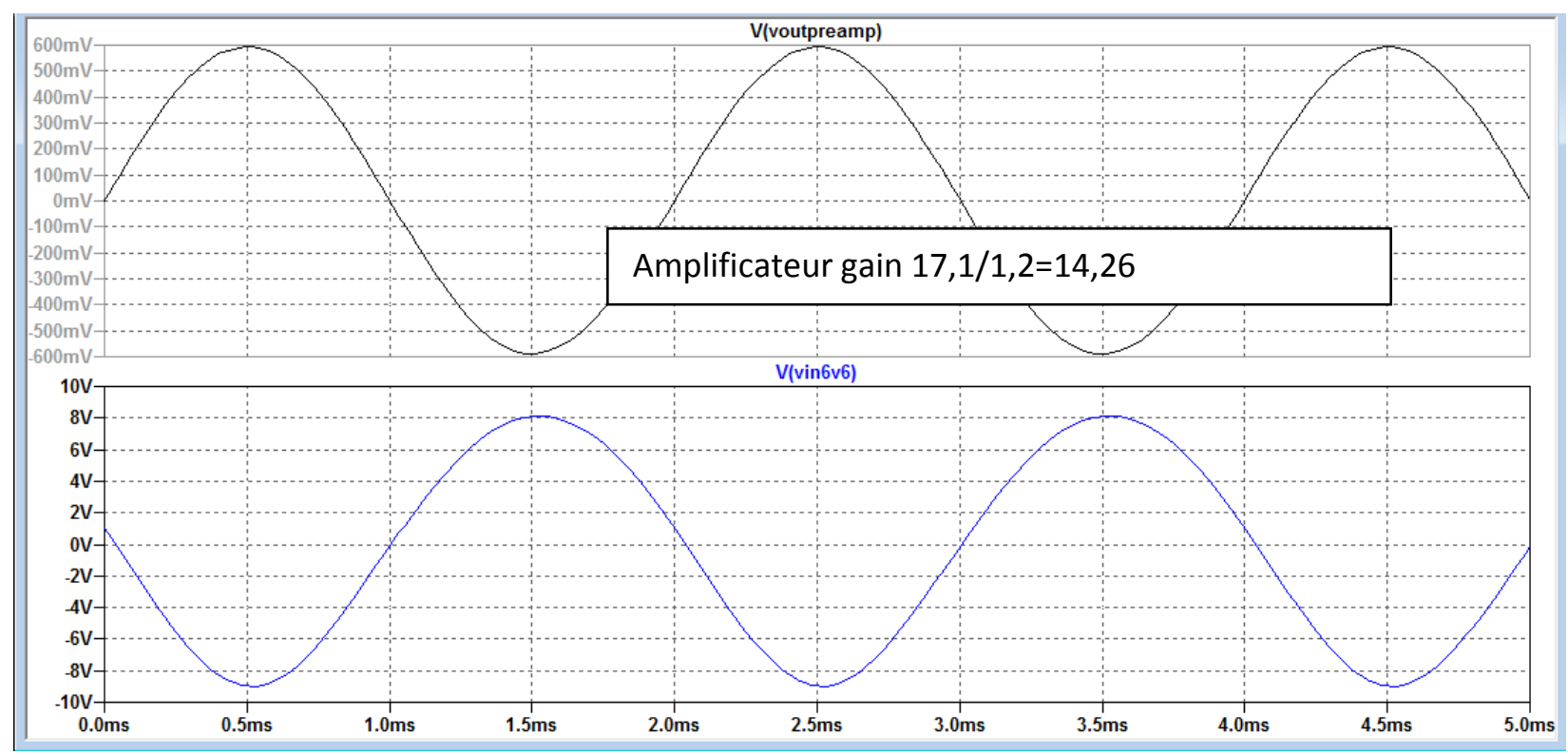

Figure 17 : Haut Entrée de l'ampli : Bas : sortie de l'ampli

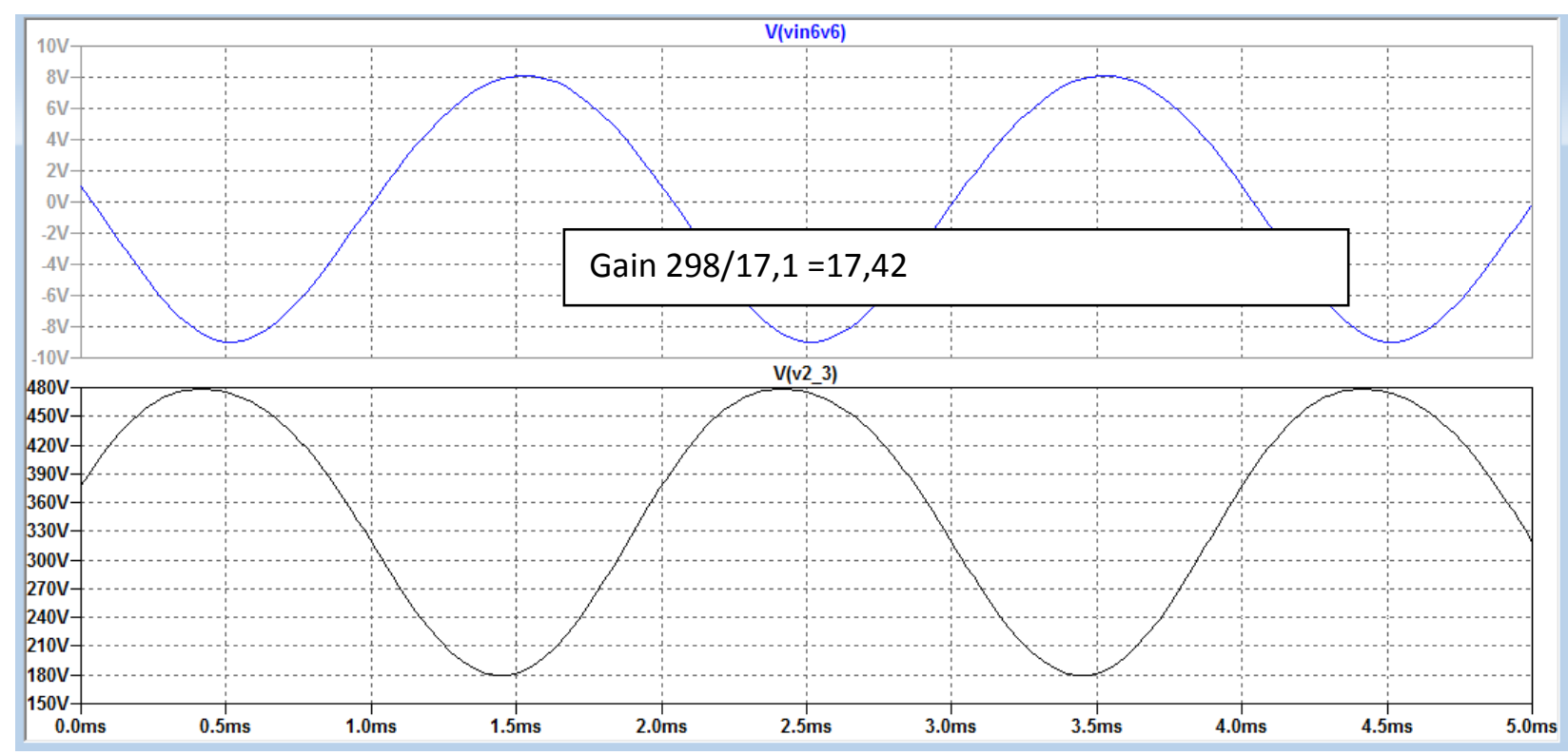

Figure 18 : Etage de puissance 6 V6 : Haut : entrée Bas : Sortie 


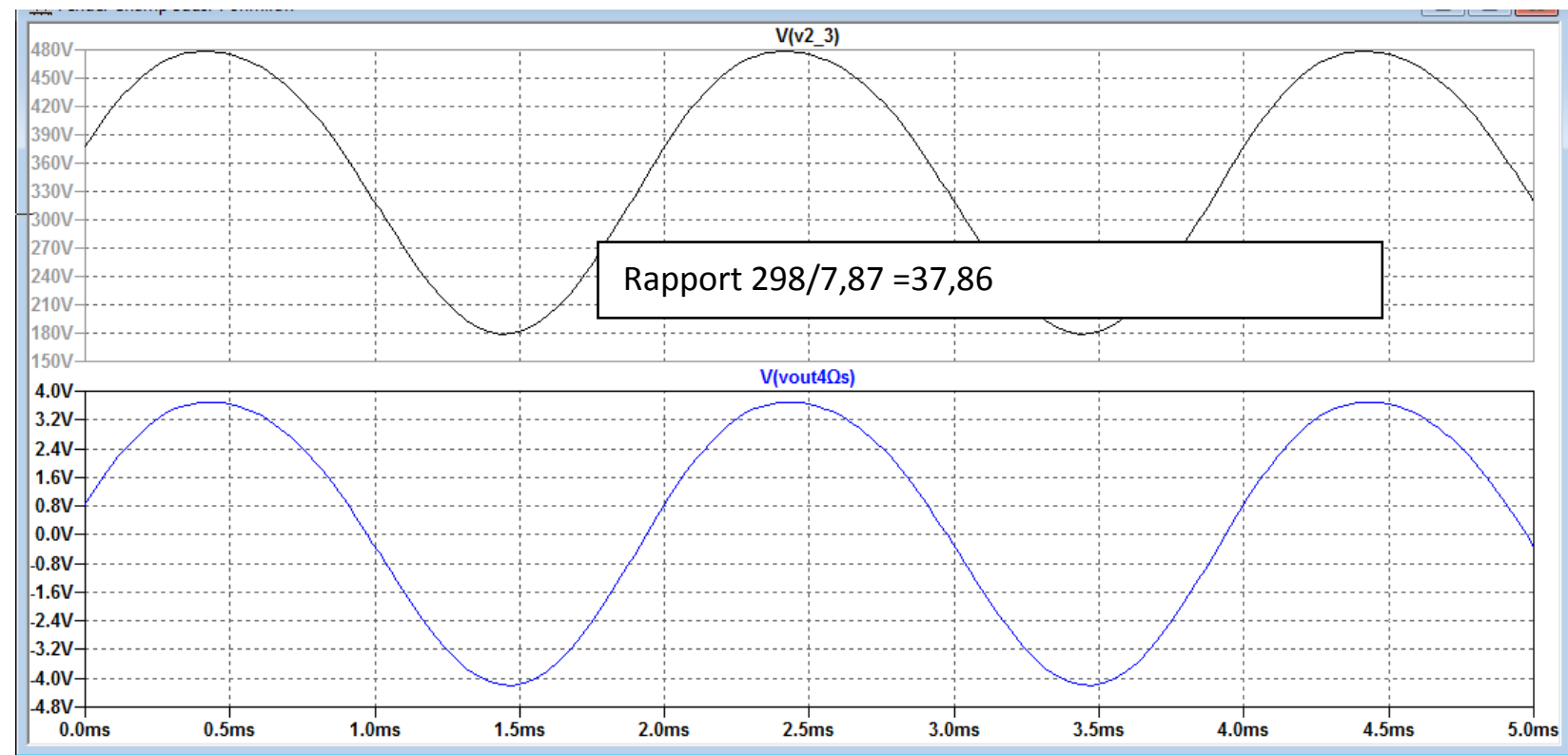

Figure 19 : Transformateur de sortie

Le mode AC leur permet de tracer la courbe de réponse en fréquence de l'amplificateur (Figure 20):

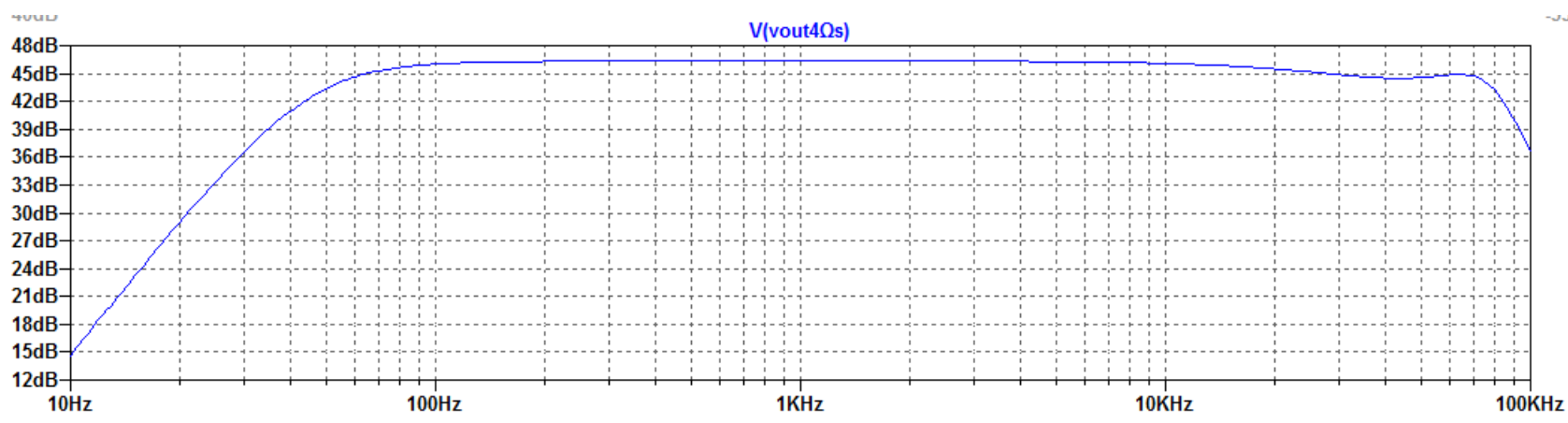

Figure 20 : Courbe de réponse en fréquence

\subsection{Montage de l'amplificateur}

Le kit approvisionné pour construire l'amplificateur contient tous les composants nécessaires à sa fabrication : les composants (résistances, condensateurs, tubes, transformateurs), la visserie, la platine, la « turret board » pour le câblage des différents éléments.

Les étudiants suivent une petite formation à la soudure et à l'utilisation des outils et matériels mis à leur disposition, cette formation est assurée par le personnel technique du département STPI de l'INSA.

Les étudiants font ensuite l'inventaire des tâches à réaliser et se répartissent les rôles. Les figures ci-dessous donnent un aperçu des différentes phases de montage : 


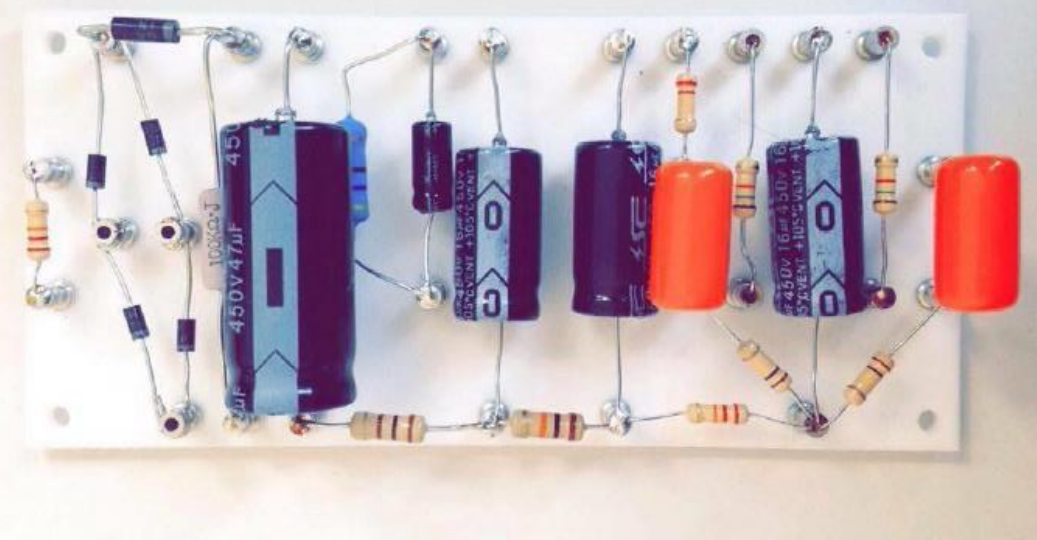

Figure 21 : La "Turret Board" avec les éléments passifs (dont le pont de diodes)

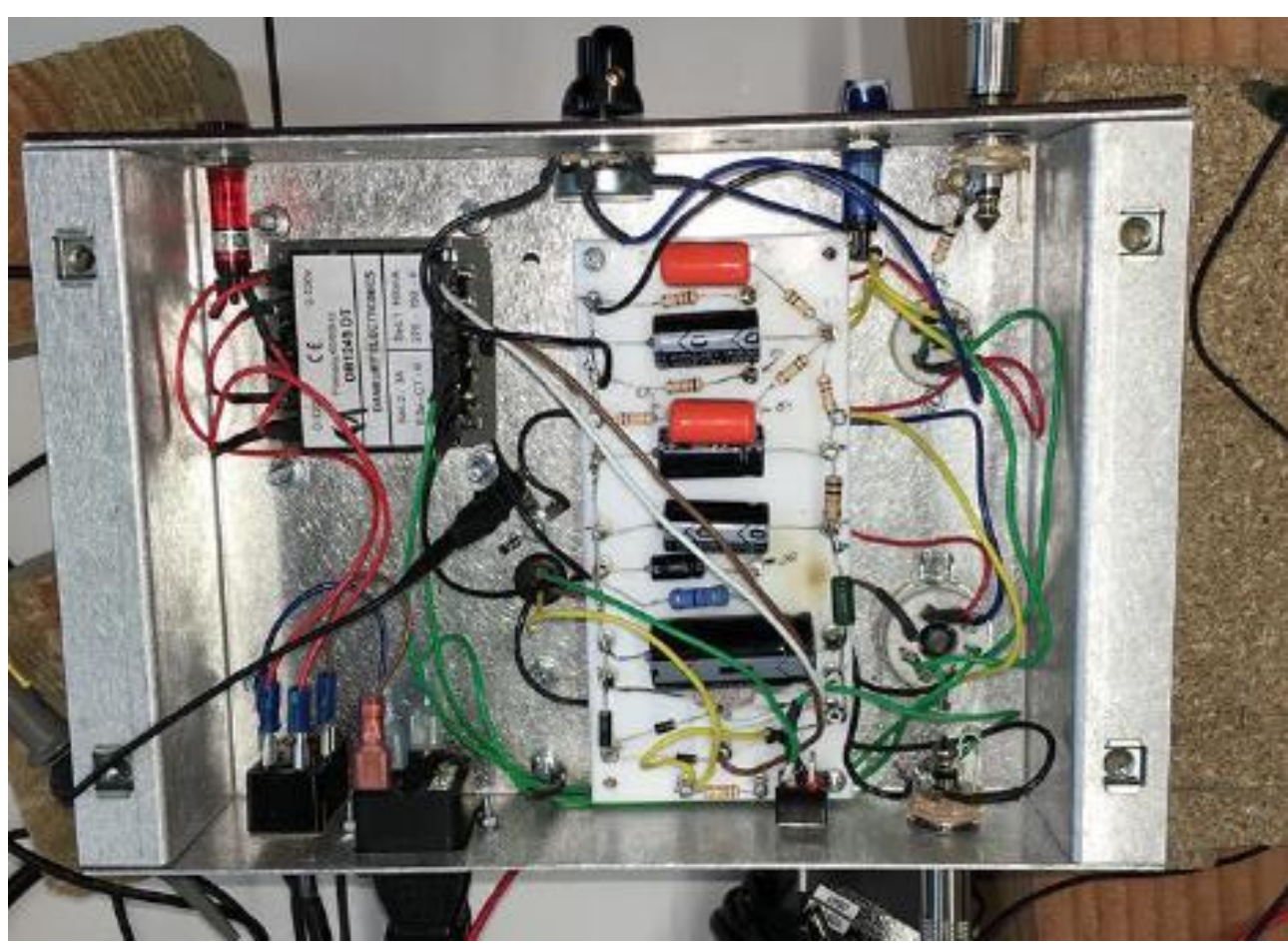

Figure 22 : Vue arrière de l'amplificateur avec le câblage 


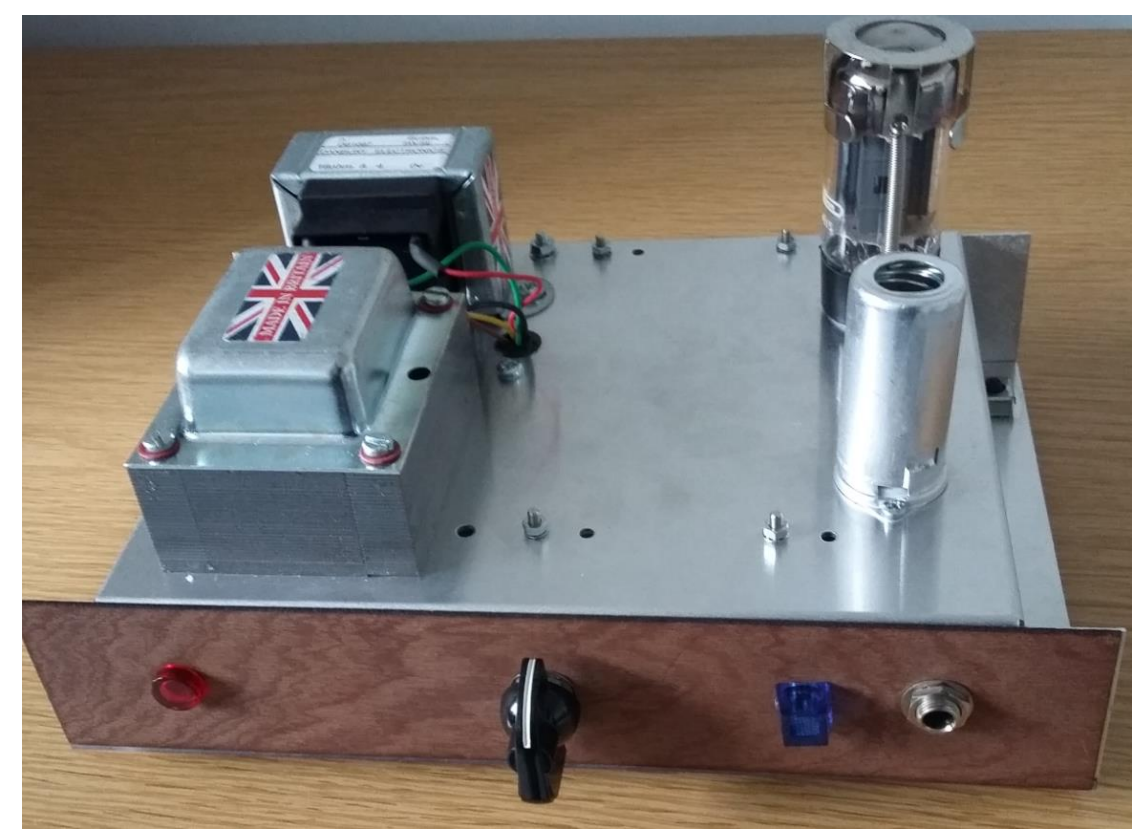

Figure 23 : L'amplificateur terminé

Par rapport au schéma d'origine il a été décidé de rajouter un commutateur permettant d'avoir une sortie en $4 \Omega$ ou en $8 \Omega$, le transformateur audio le permettant.

\subsection{Les mesures et tests}

Pour effectuer les mesures sans faire de bruit, l'amplificateur a été chargé par une résistance de puissance de $4 \Omega$. Les Figures 24 à 27 permettent de constater qu'il y a de faibles écarts entre les calculs, la simulation et les mesures (effectuées ici à $500 \mathrm{~Hz}$ et $20 \mathrm{mVPP}$ en entrée).

Le calcul pratique du gain de l'étage d'entrée à l'oscilloscope donne 552/20,8 soit 26,54 comparé à 30 théorique (Figure 25 et Figure 16). Le calcul du gain du deuxième étage donne 8,6/0,592 soit 14,52 comparé à 14,26 (Figure 26 et Figure 17). Le calcul du gain du 6V6 donne 164/9=18,22 comparé à 17,42 (Figure 27 et Figure 18).

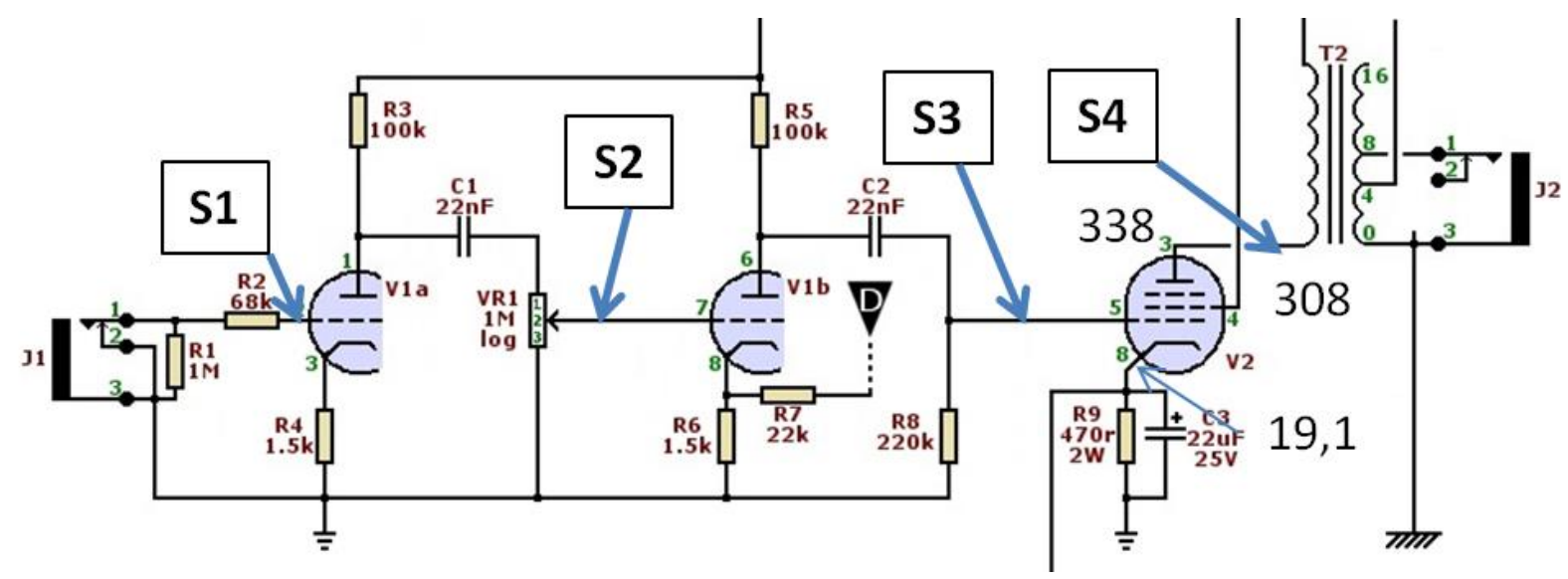

Figure 24 : Placement des sondes oscilloscopes (points S1, S2, S3, S4) 

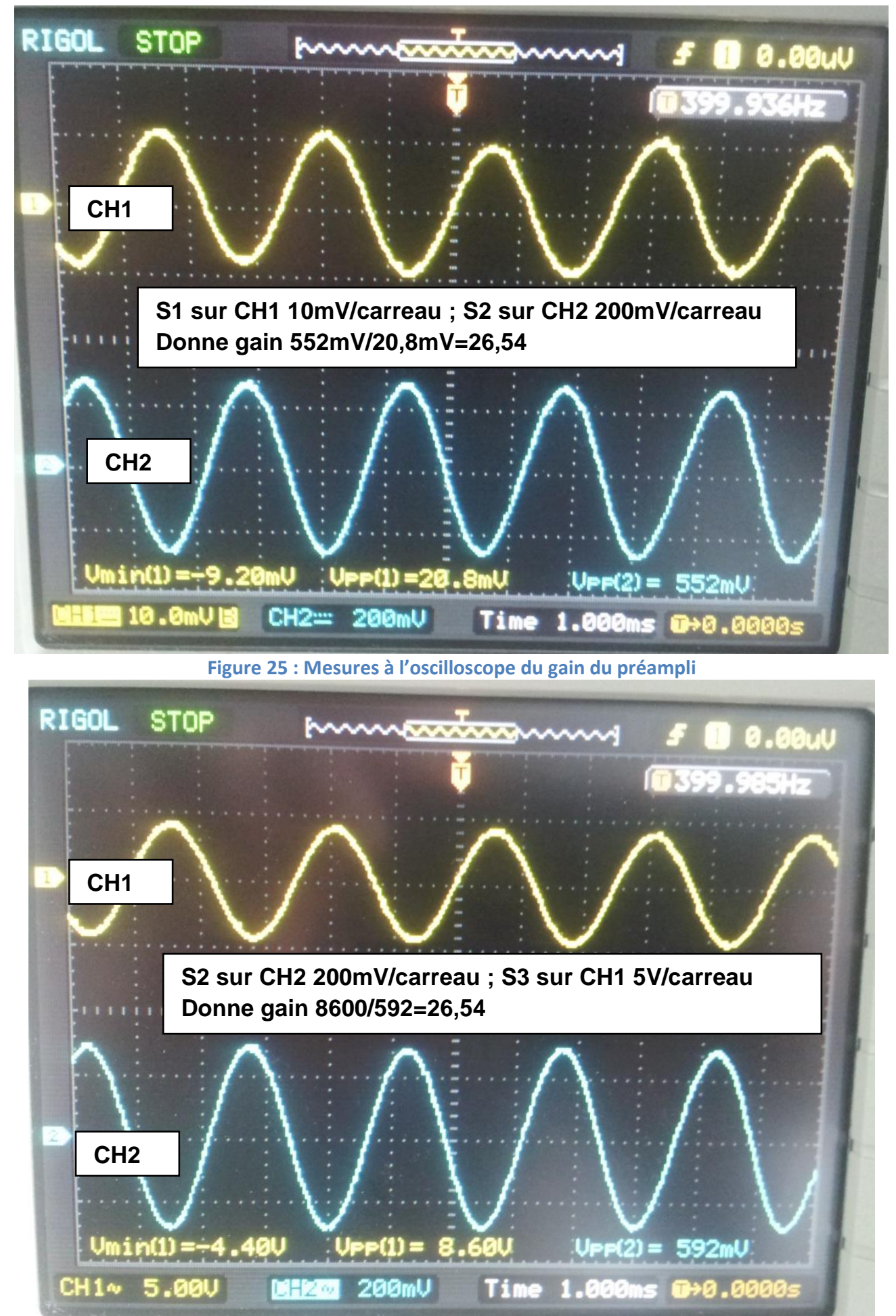

Figure 26 : Mesures à l'oscilloscope du gain du deuxième étage 


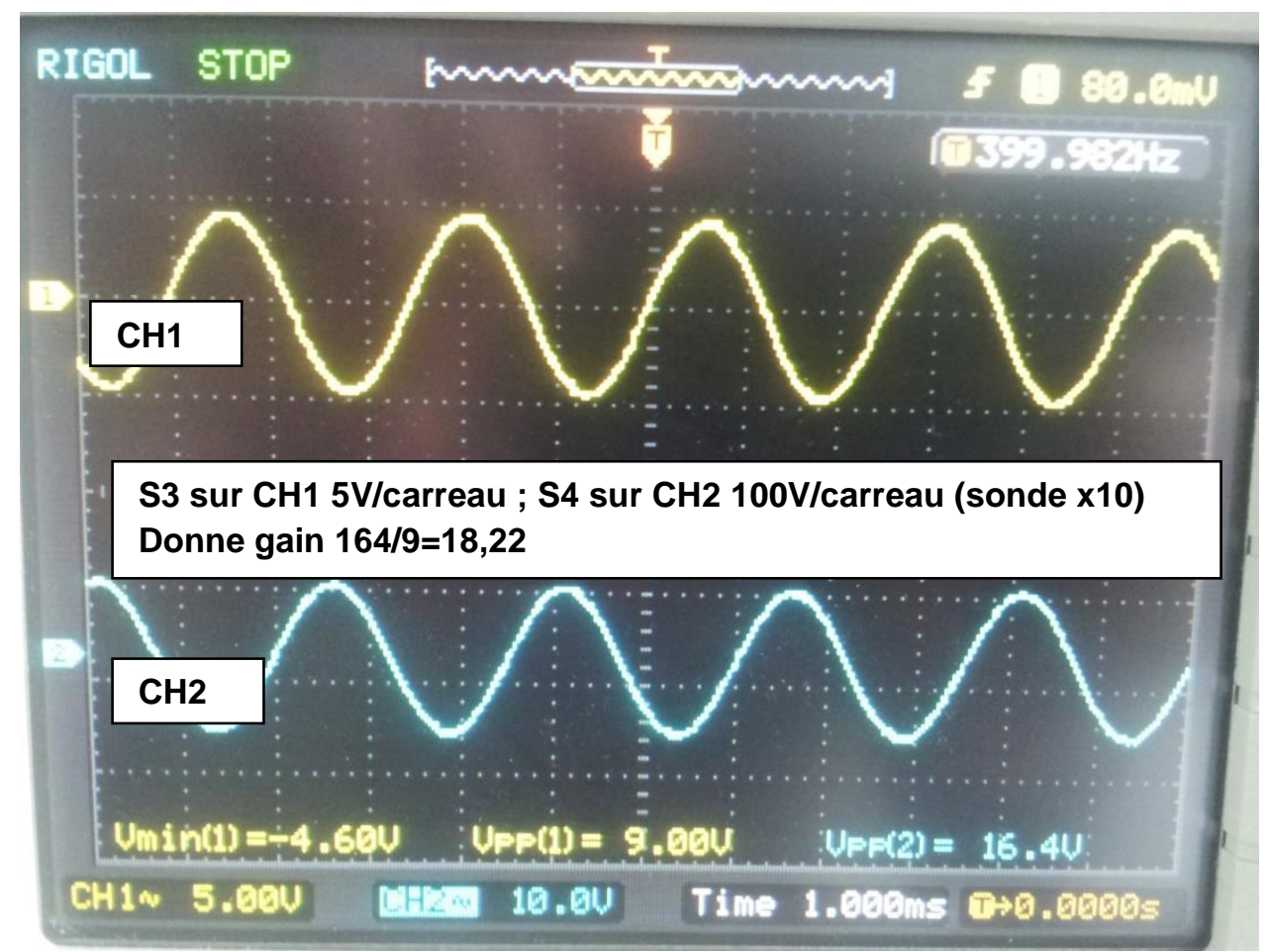

Figure 27 : Mesures à l'oscilloscope du gain de l'étage de sortie

\subsection{Evolution du montage et mise en boitier}

Il a été ensuite décidé d'ajouter un circuit de tonalité, le plus simple possible, une recherche dans les documentations Fender a fait apparaître la version modifié du Fender Champ (Tweed ou Princeton) avec un circuit de tonalité simple à un seul potentiomètre. 


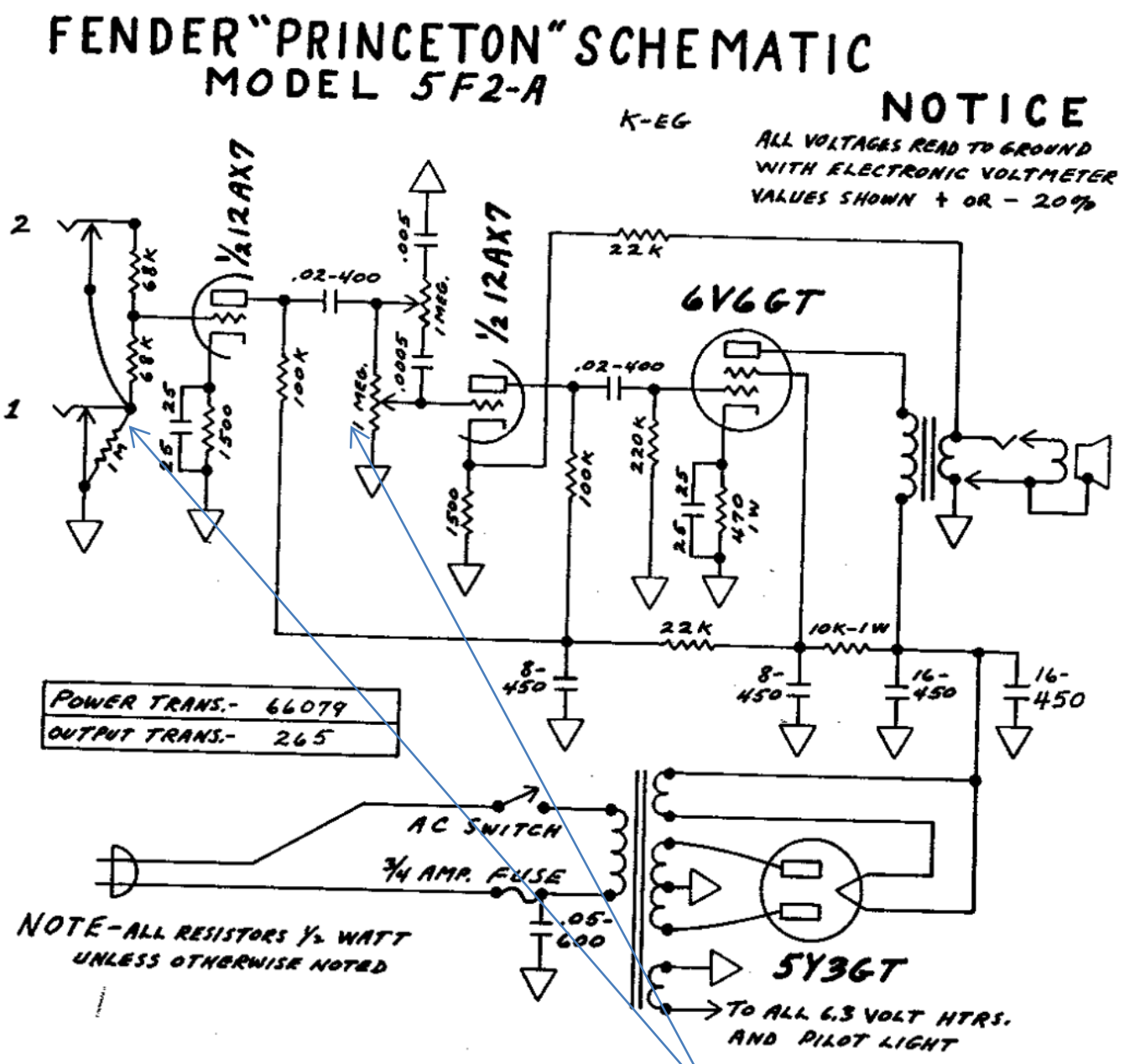

Figure 25 : Schéma de la version Fender avec potentiomètre de tonalité

L'étude de la modification du schéma permet de voir l'effet de l'ajout du potentiomètre de tonalité (Figures 28, 29 et 30).

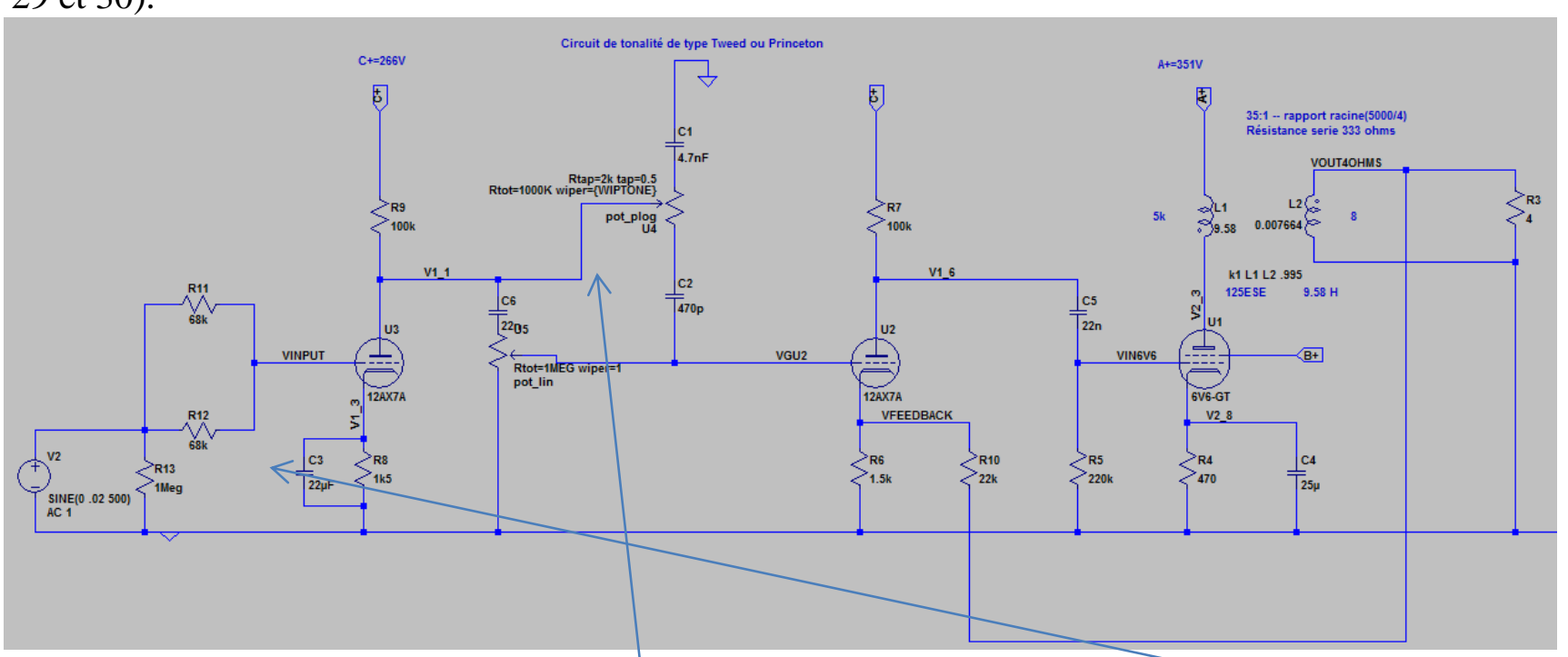

Figure 26 : Ajout du circuit de tonalité type TWEED ou PRINCETON et du condensateur sur le préampli 


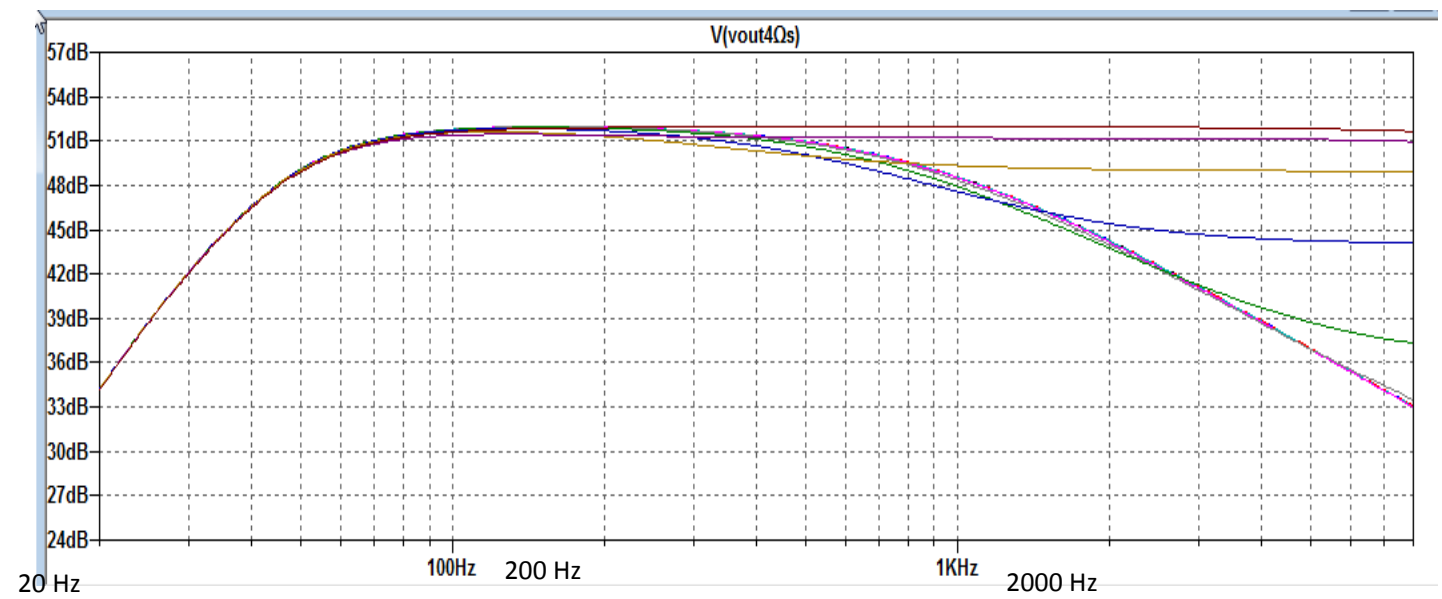

Réponse fréquentielle expérimentale de l'amplificateur en fonction du réglage de la tonalité

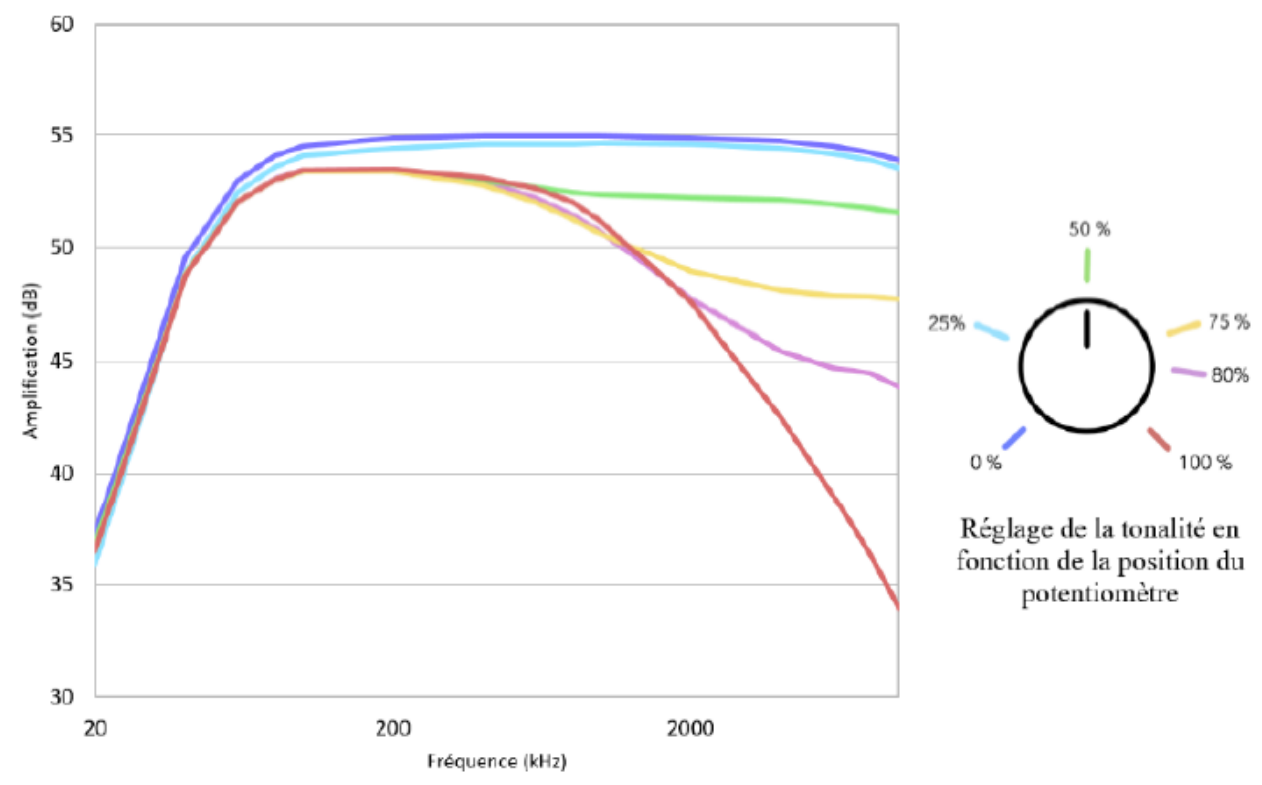

Figure 27 : Courbe de réponse avec circuit de tonalité (haut : LTSPICE, bas : relevé expérimental)

On constate l'effet de type coupure des hautes fréquences du circuit de tonalité, la perte de gain occasionné par l'ajout du potentiomètre de tonalité est compensée par l'ajout d'un condensateur de court-circuit sur la résistance de polarisation du premier étage d'amplification ce qui fait passer son gain à 60 au lieu de 30 .

Les étudiants se sont ensuite occupés de mettre en boitier l'amplificateur et d'intégrer un haut-parleur de 20 watts dans un caisson prévu pour cela ce qui donne l'aspect final de l'amplificateur de manière à ce qu'il puisse être utilisé et transporté facilement. 


\section{Présentation des caractéristiques du VOX AC-30 et approche pédagogique}

La figure 31 présente le schéma original du VOX AC-30, la figure 32 la version simplifiée faisant apparaître les différentes parties de l'amplificateur.

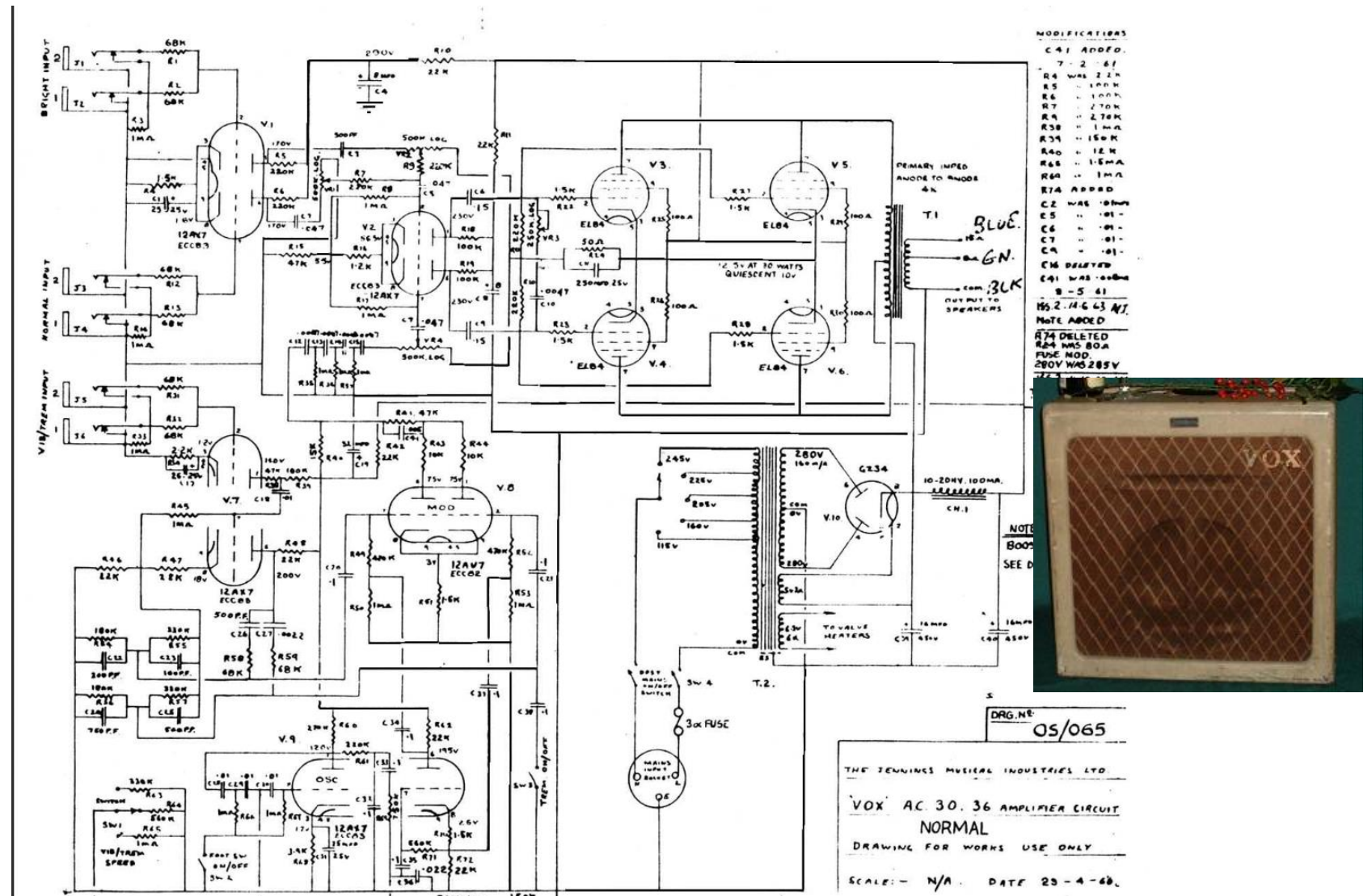

Figure 28 : Schéma original et photographie du VOX AC-30

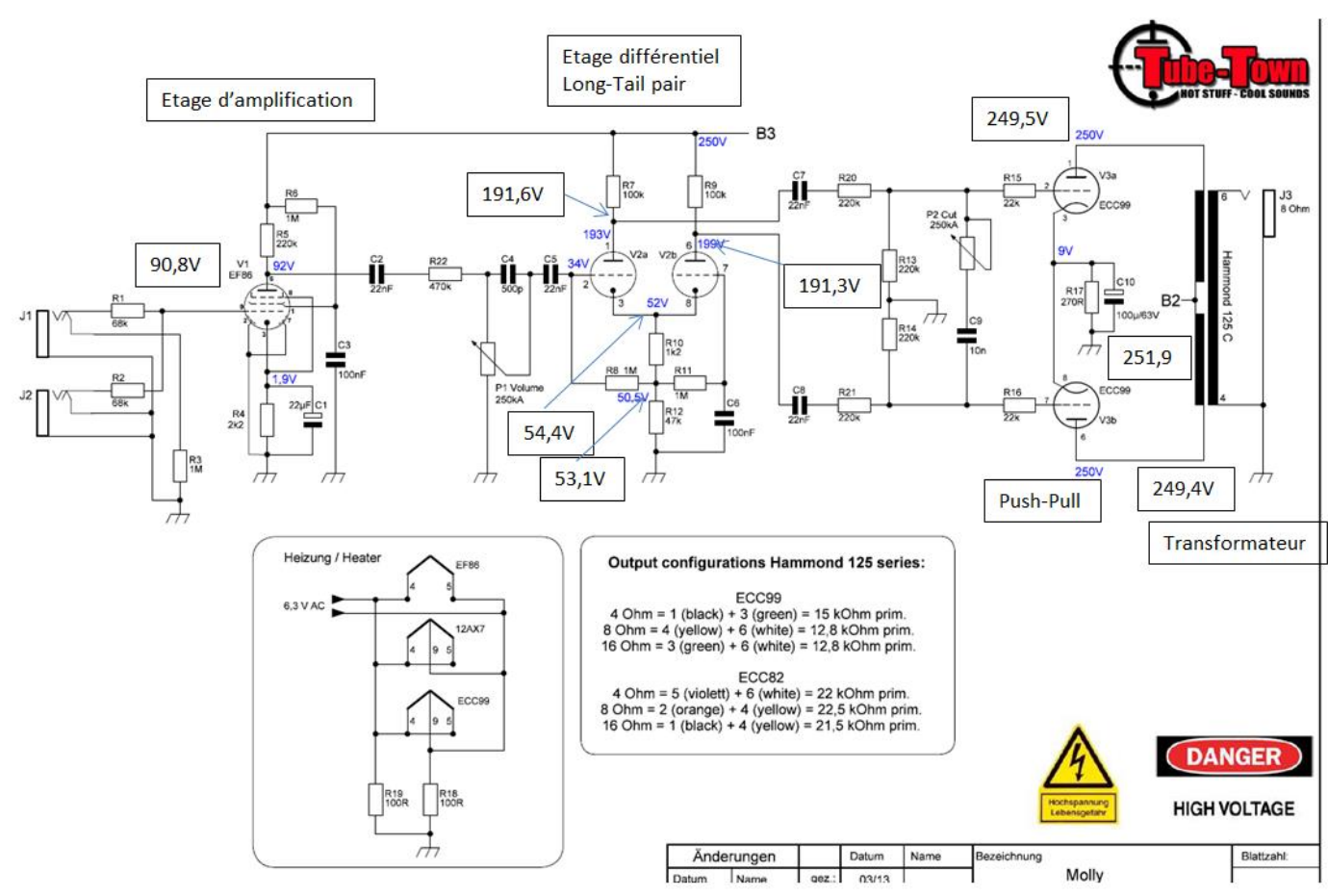

Figure 29 : Version du Kit AC-30 low-power

Il faut noter aussi que l'alimentation est construite sur un circuit imprimé double face dont le schéma est donné sur la figure 33 : 


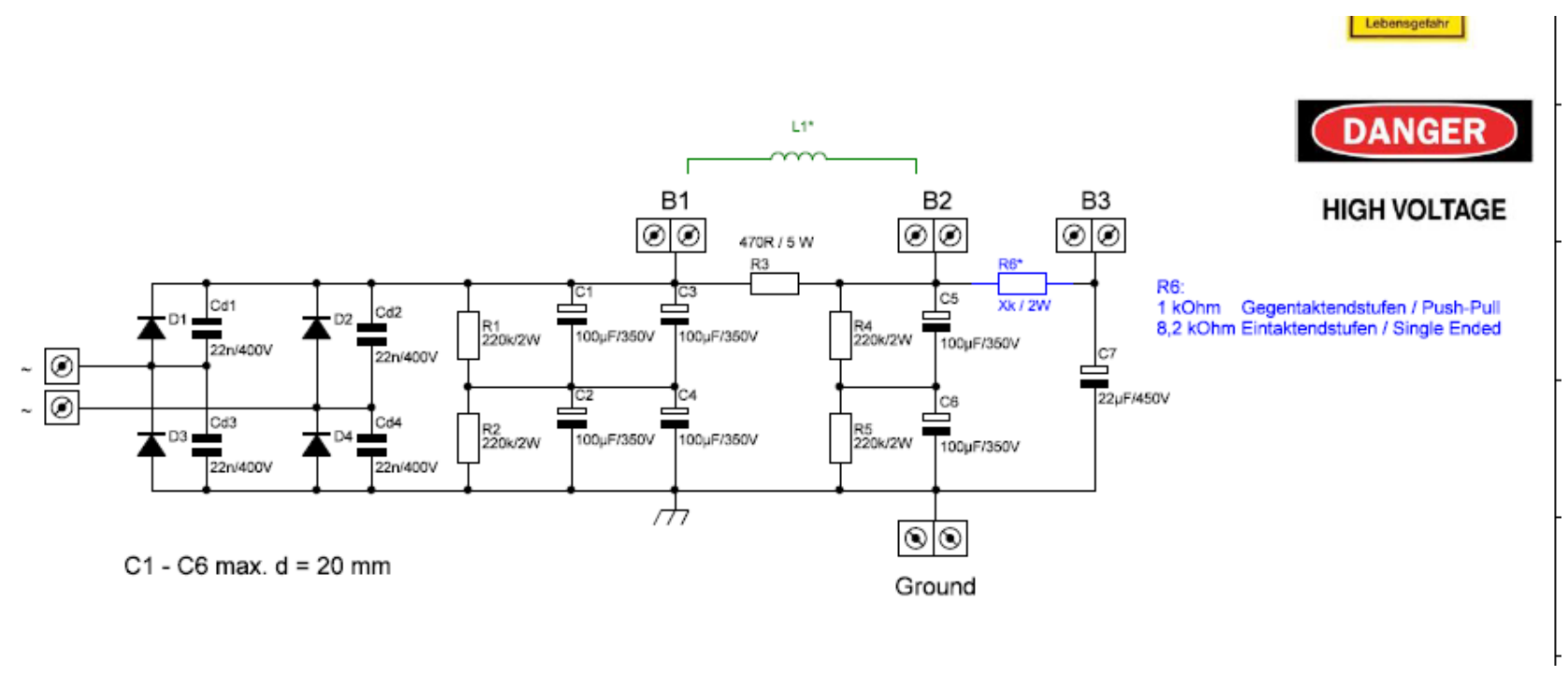

Figure 30 : Alimentation du VOX-AC30

\subsection{Etude de l'étage d'amplification}

Le préampli correspond au schéma suivant :

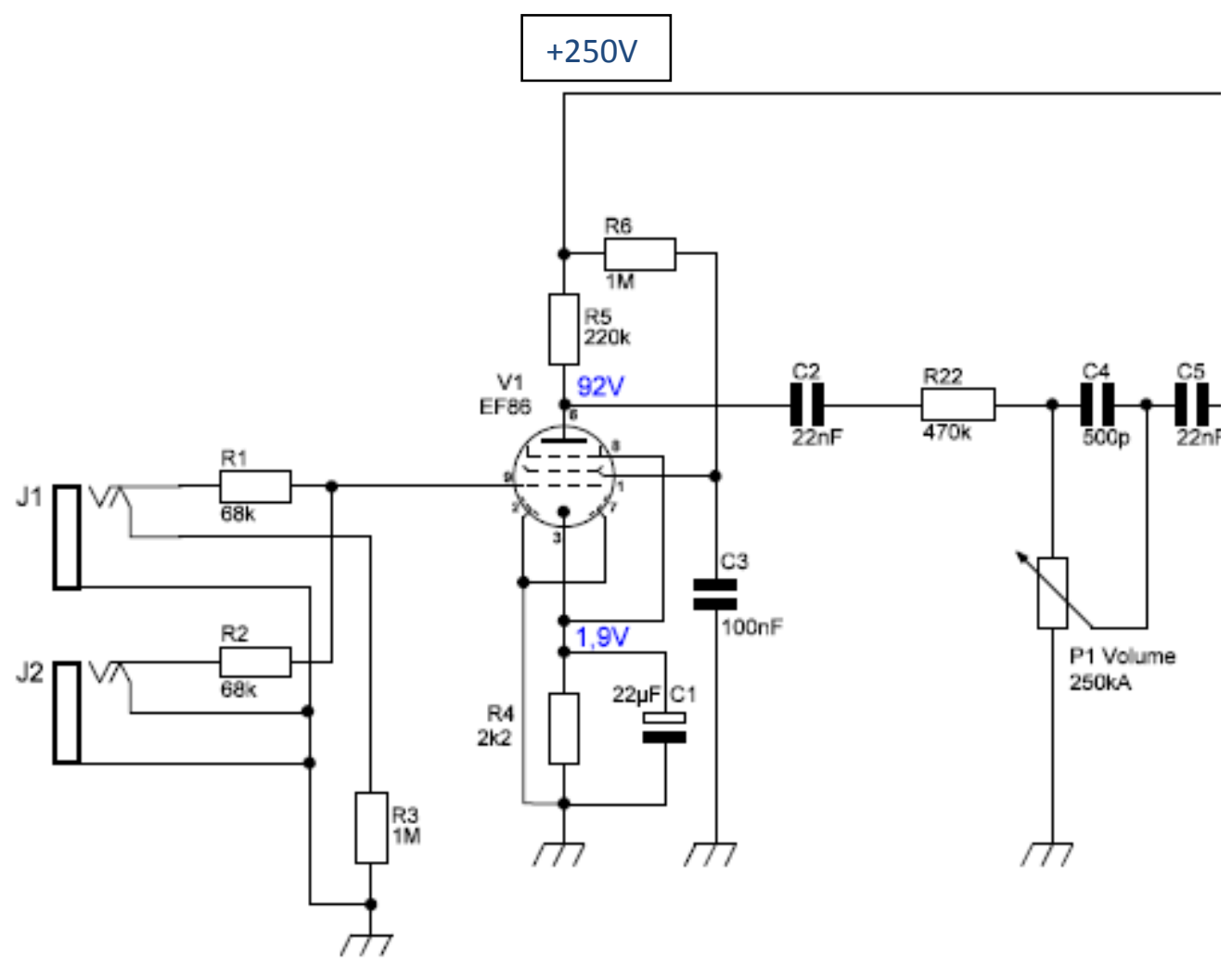

Figure 31 : Etage d'entrée du VOX AC-30

Il s'agit d'un amplificateur avec une pentode, c'est l'étage d'entrée des premiers VOX (VOX AC30 et VOX $\mathrm{AC} 15)$, il est donc largement documenté dans la littérature des circuits à lampe, on trouve notamment à la page 48 de la référence [6] le schéma et le schéma équivalent en petits signaux: 


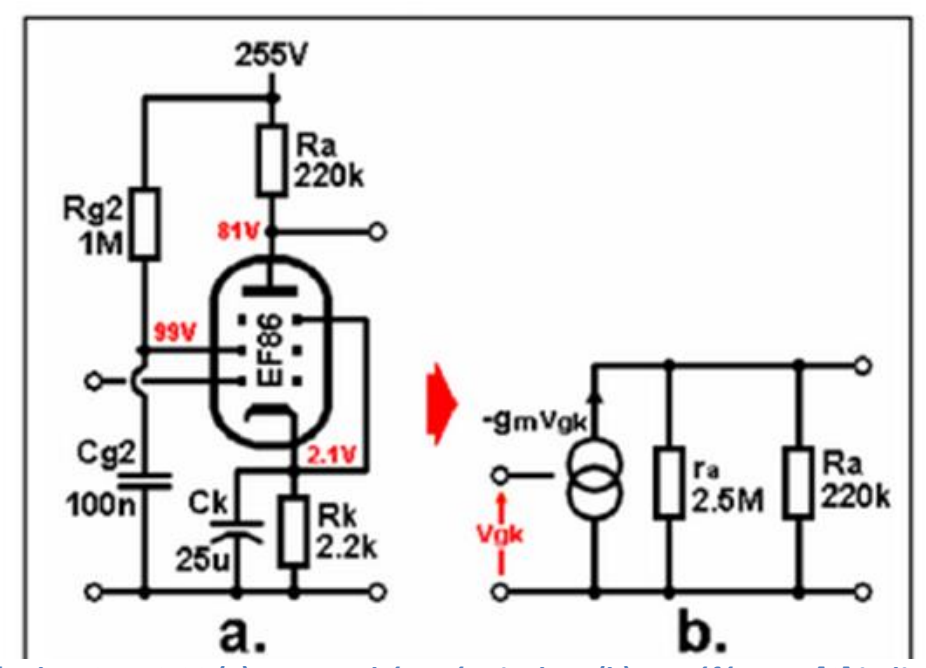

Figure 32 : Etage d'entrée du VOX AC-30 (a) et son schéma équivalent (b). La référence [6] indique un gain d’environ 200

Il est expliqué aux étudiants que la résistance de charge doit être remplacée par la $220 \mathrm{k} \Omega$ en parallèle avec la résistance de charge de l'amplificateur qui suit la pentode soit pour nous $220 \mathrm{k} \Omega / /(470 \mathrm{k} \Omega+250 \mathrm{k} \Omega)$ soit $(220 \mathrm{k} \Omega / / 720 \mathrm{k} \Omega)=168 \mathrm{k} \Omega$. La documentation Philips EF86 [7] et la littérature donnent une valeur de la transconductance gm d'environ $0,95 \mathrm{~mA} / \mathrm{V}$ pour ce montage.

Le calcul du gain de l'étage donne donc en première approximation:

$$
\mathrm{Av}=-\mathrm{g}_{\mathrm{m}} * \mathrm{R}_{\mathrm{L}}\left(\operatorname{avec} \mathrm{R}_{\mathrm{L}}=(220 \mathrm{k} \Omega / / 720 \mathrm{k} \Omega)\right)=0,95 * 168=159,6
$$

\subsection{Etude de l'amplificateur différentiel}

L'explication donnée aux étudiants concernant ce montage (Figure 36) est très simple, il leur est expliqué qu'il permet de fournir en sortie 2 signaux en opposition de phase.

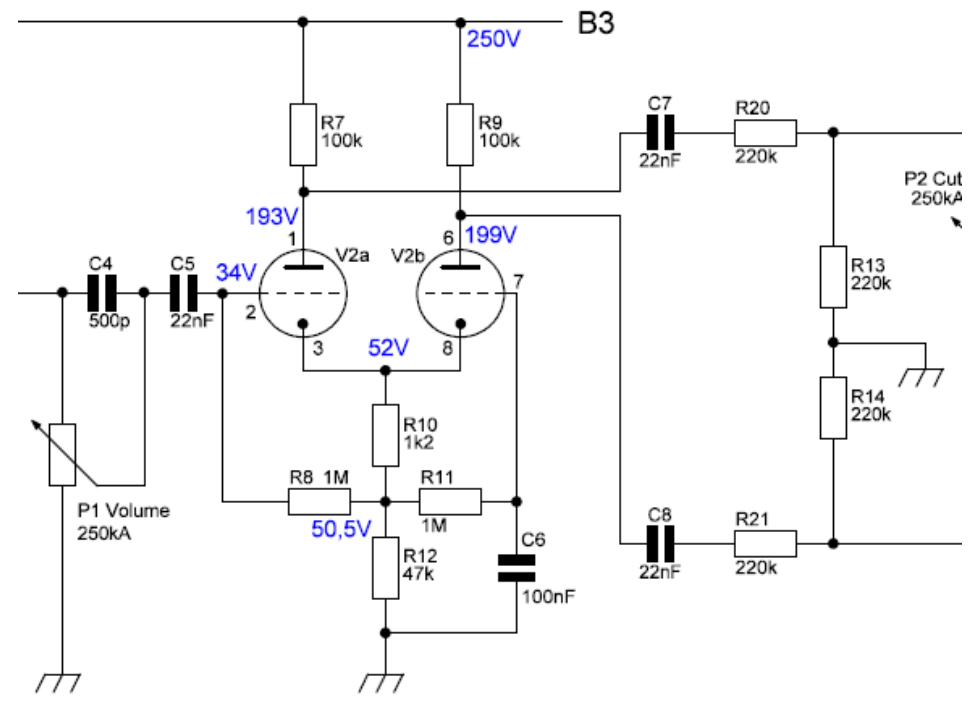

Figure 33 : Etage différentiel encore appelé "Long Tailed pair"

Pour les calculs on leur donne la référence d'un calculateur en ligne, avec les éléments de la figure 37 https://www.ampbooks.com/mobile/amplifier-calculators/long-tailed-pair/ 


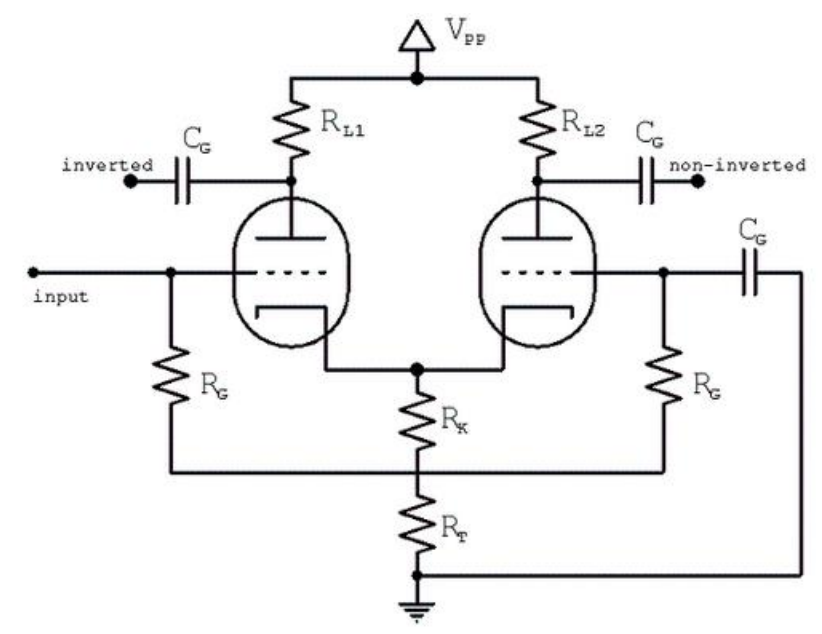

Figure 34 : repérage des éléments pour le calculateur en ligne

Les résistances $\mathrm{R}_{\mathrm{L} 1}$ et $\mathrm{R}_{\mathrm{L} 2}$ en mode petits signaux sont données à approximativement $81 \mathrm{k} \Omega$ (soit $100 \mathrm{k} \Omega\left(\mathrm{R}_{7}\right.$ ou $\left.\mathrm{R}_{9}\right) / /(220 \mathrm{k} \Omega+220 \mathrm{k} \Omega)\left(\mathrm{R}_{20}+\mathrm{R}_{13}\right.$ ou $\left.\mathrm{R}_{21}+\mathrm{R}_{14}\right)$, ce calcul n'est pas tout à fait exact car il faudrait aussi tenir compte de la résistance du potentiomètre de tonalité, cela donne:

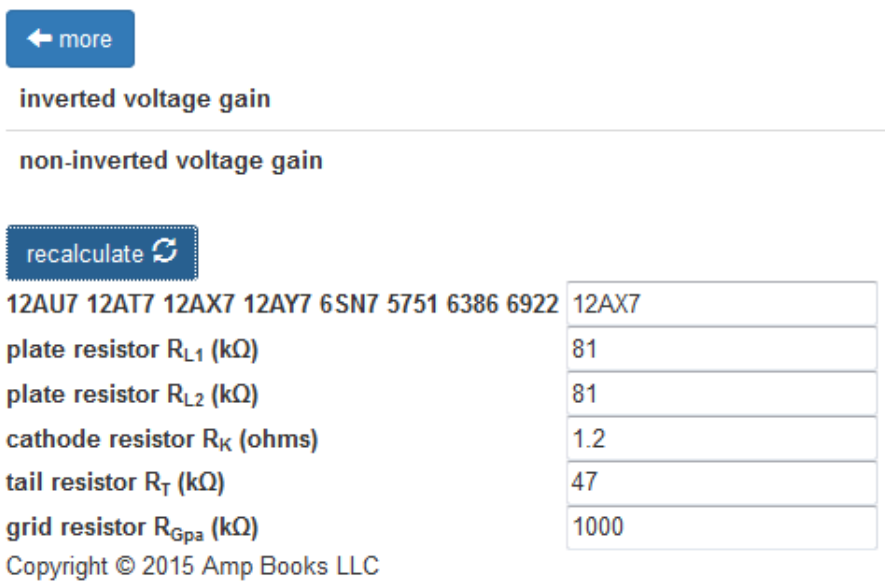

Pour les étudiants qui veulent faire le calcul avec les droites de charge qui ont été présentées dans l'introduction on leur donne la référence du site http://www.valvewizard.co.uk/acltp.html

Ce qui donne les paramètres de calcul suivants, sachant que l'on a des 12AX7.

- Courant total : $1 \mathrm{~mA}$ soit un courant dans chaque tube de $0,5 \mathrm{~mA}$

- Tension d'alimentation : $250 \mathrm{~V}$

- Tension à l'anode : $192 \mathrm{~V}\left(0,58 \mathrm{~mA}\right.$ dans $\mathrm{R}_{7}$ ou $\mathrm{R}_{9}$ de $100 \mathrm{k} \Omega$ donne une chute de tension de $\left.58 \mathrm{~V}\right)$

- Tension aux bornes de $\mathrm{R}_{t}: 53,1 \mathrm{~V}$ (au lieu de 52V sur la figure 32)

- Tension aux bornes de $\mathrm{R}_{\mathrm{k}}$ de $1,2 \mathrm{~V}$ (aux bornes de $\mathrm{R}_{10}$, figure 32 )

- Tension $\mathrm{V}_{\mathrm{gk}}$ de polarisation $=1,2 * 1 \mathrm{~mA}$ soit environ $1,2 \mathrm{~V}\left(\mathrm{R}_{\mathrm{k}} \cdot \mathrm{I}_{\text {atotal }}\right)$

- On calcule : $\left(53,1+1,2\right.$ donne 54,4 pour la différence avec $\left.\mathrm{V}_{\mathrm{a}}\right)$ $\mathrm{V}_{\mathrm{p}}$ point de polarisation $=192-54,4=137,6 \mathrm{~V} .1,3 \leq \mathrm{V}_{\mathrm{k}} \leq 1,4$ $\mathrm{V}_{\mathrm{A}}$ (droite de charge) polarisation $=250$ - $\left(\right.$ tension aux bornes de $\left.\mathrm{R}_{\mathrm{T}}\right)=250-53,1=196,9 \mathrm{~V}$

Sur les grilles de la 12AX7 « data sheet » cela donne (Figure 38), sur le modèle du paragraphe 3.1 : 
MESURES : Point de polarisation 12AX7.

La tension de plate est diminuée de la tension aux bornes de la 47k (R12 sur le schéma figure 32)

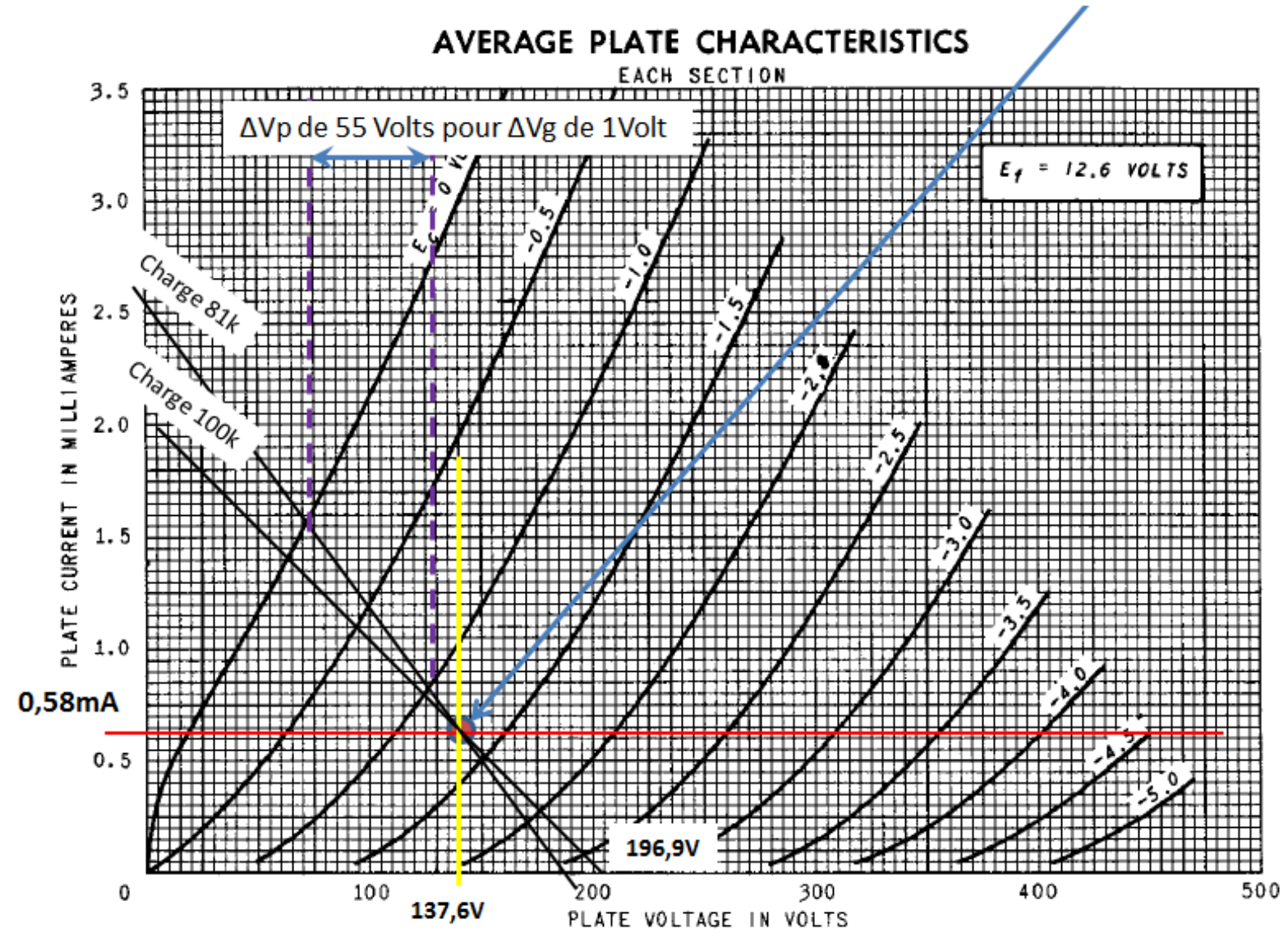

Figure 35 : Polarisation graphique pour l'amplificateur différentiel

La droite de charge en continu passe par le point $(0 \mathrm{~mA}-196,9 \mathrm{~V})$ et le point de fonctionnement $(0,58 \mathrm{~mA}$ $137,6 \mathrm{~V})$. Le gain se calcule à partir de la droite de charge en dynamique (charge de $81 \mathrm{k} \Omega$ ), pour une augmentation de $\mathrm{V}_{\mathrm{g}}$ de $1 \mathrm{~V}$ cela donne $55 \mathrm{~V}$ soit un gain théorique par étage de 27,5 car le gain se répartit pour moitié entre les deux lampes.

\subsection{Etude du « Push-Pull » de sortie (triode ECC99)}

La figure 39 donne le schéma de l'étage de sortie de type «push-pull ». 


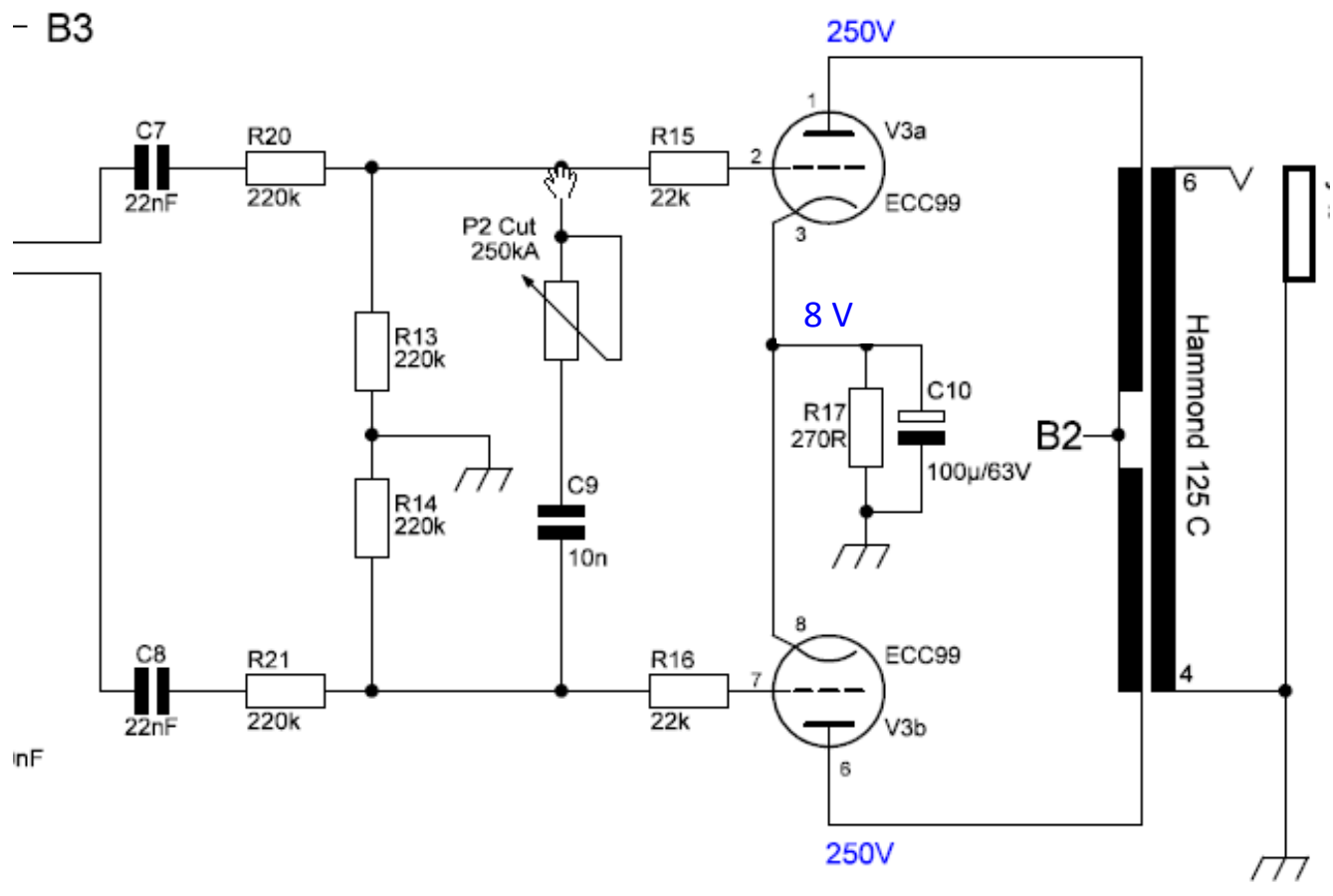

Figure 36 : Etage de sortie et transformateur

On explique aux étudiants que la charge étant un transformateur supposé parfait sa résistance statique est nulle, on retrouve donc intégralement les $250 \mathrm{~V}$ du point B2 sur les anodes des triodes.

Pour la charge de 4 ohms, on voit aux bornes $\mathrm{V}_{3 \mathrm{a}}$ et $\mathrm{V}_{3 \mathrm{~b}}$ une résistance de charge dynamique égale à :

$$
4 *\left(\frac{N 1}{N 2}\right)^{2} * R L=R p p=15000 \Omega
$$

La documentation de 1'EC99[8] donne

$$
\begin{aligned}
& \text { TYPICAL } \\
& U_{\mathrm{a}}=150 \mathrm{~V} \\
& \mathrm{~S}=9,5 \mathrm{~mA} / \mathrm{V} \\
& \text { CHARACTERISTIC: } \\
& \mathrm{U}_{\mathrm{g}}=-4 \quad \mathrm{~V} \\
& R_{i}=2,3 \quad k \Omega \\
& \mathrm{l}_{\mathrm{a}}=18 \mathrm{~mA} \\
& \mu=22
\end{aligned}
$$

On peut donc reprendre le calcul théorique du gain du montage pour chacun des demi-étages du PUSHPULL (voir calcul du préampli du Fender au paragraphe 3.1) par le schéma équivalent suivant :

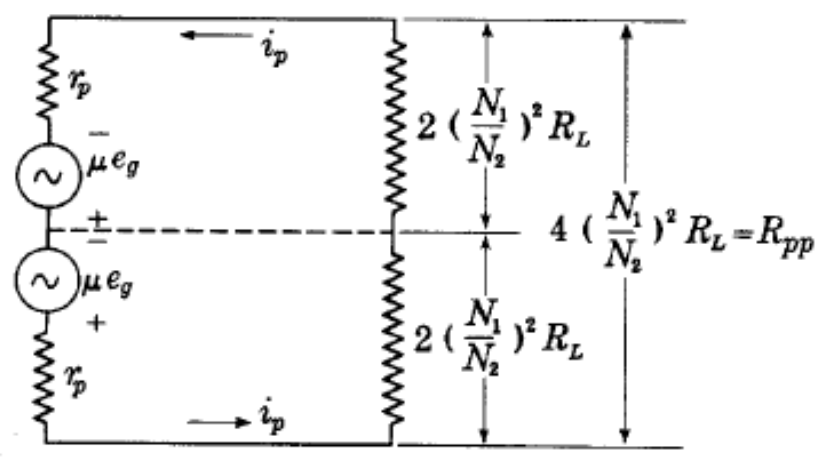

(a)

Figure 37 : Schéma équivalent 
Chaque triode voit donc une résistance dynamique égale à $7500 \Omega$. Ce qui permet de tracer la droite de charge comme précédemment expliqué pour une 12AX7 (Figure 41):

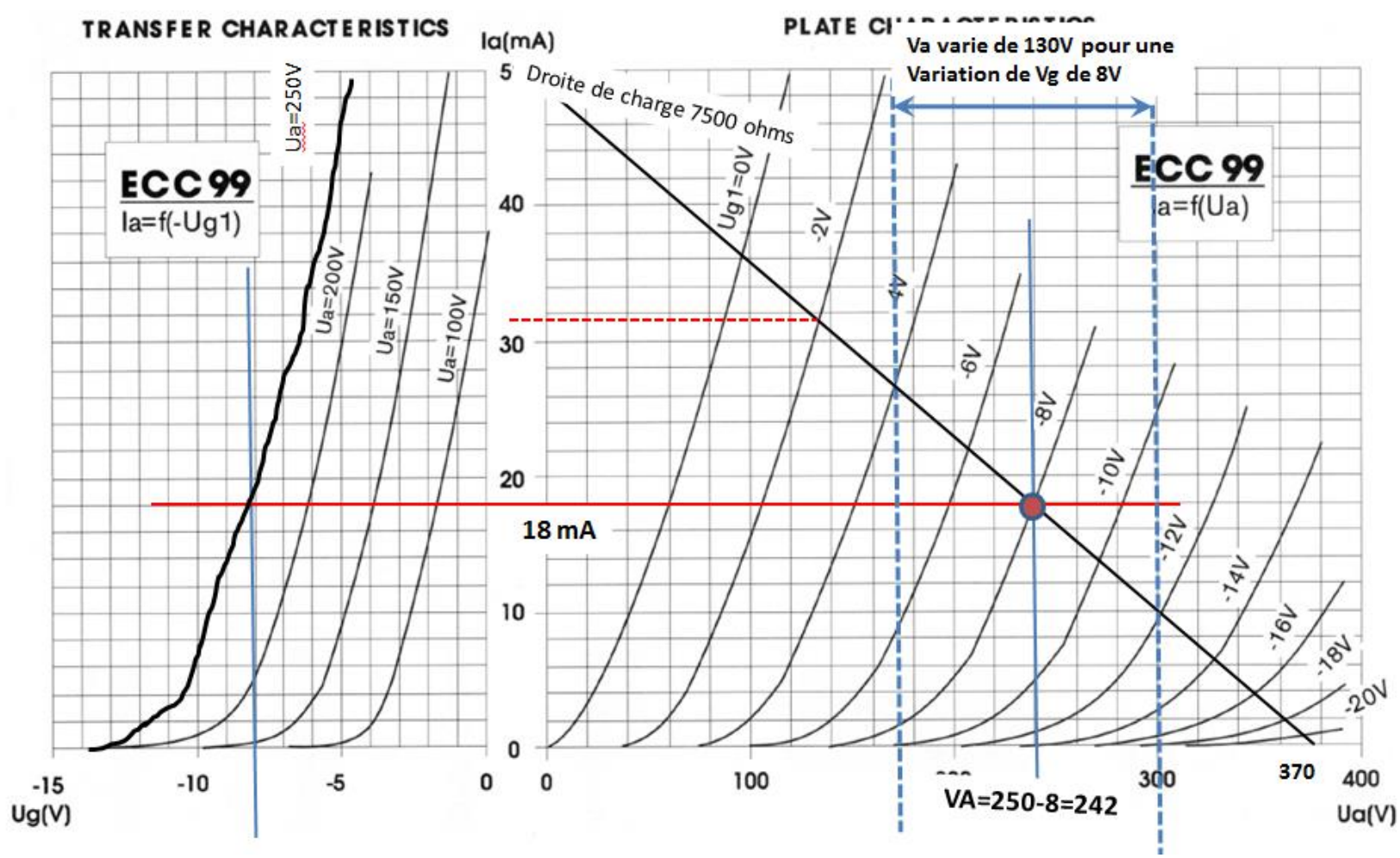

Figure 38 : Droites de charge pour l'étage de sortie sur 4 ohms

Le gain par étage (calcul graphique) est donc de 130/8 soit 16,25. On constate que l'excursion maximale positive (donc quand l'alternance d'entrée est négative) est de (370-242)=128V au grand maximum (avec distorsion dans le haut) donc une valeur maximale d'entrée de 128/16,25=7,9V.

Contrairement à l'ampli Fender, le transformateur utilisé a une résistance dynamique qui n'est pas la même pour une sortie sur un haut-parleur de $8 \Omega$ :

\section{Output configurations Hammond 125 series:}

\section{ECC99}

$4 \mathrm{Ohm}=1$ (black) +3 (green) $=15 \mathrm{kOhm}$ prim.

$8 \mathrm{Ohm}=4$ (yellow) +6 (white) $=12,8 \mathrm{kOhm}$ prim .

$16 \mathrm{Ohm}=3$ (green) +6 (white) $=12,8 \mathrm{kOhm}$ prim.

Donc $\left(\frac{2 . N 1}{N 2}\right)^{2}=\frac{12800}{8}=\frac{R p p}{R L}$. La résistance dynamique passe donc à $12800 \Omega$.

Le point de polarisation est toujours à $(18 \mathrm{~mA} ; 242 \mathrm{~V})$ mais la droite de charge change $(6400 \Omega)$. Cela donne une variation de $160 \mathrm{~V}$ pour une variation $\mathrm{V}_{\mathrm{g}}$ de $10 \mathrm{~V}$, soit un gain de 16 (Figure 42) : 


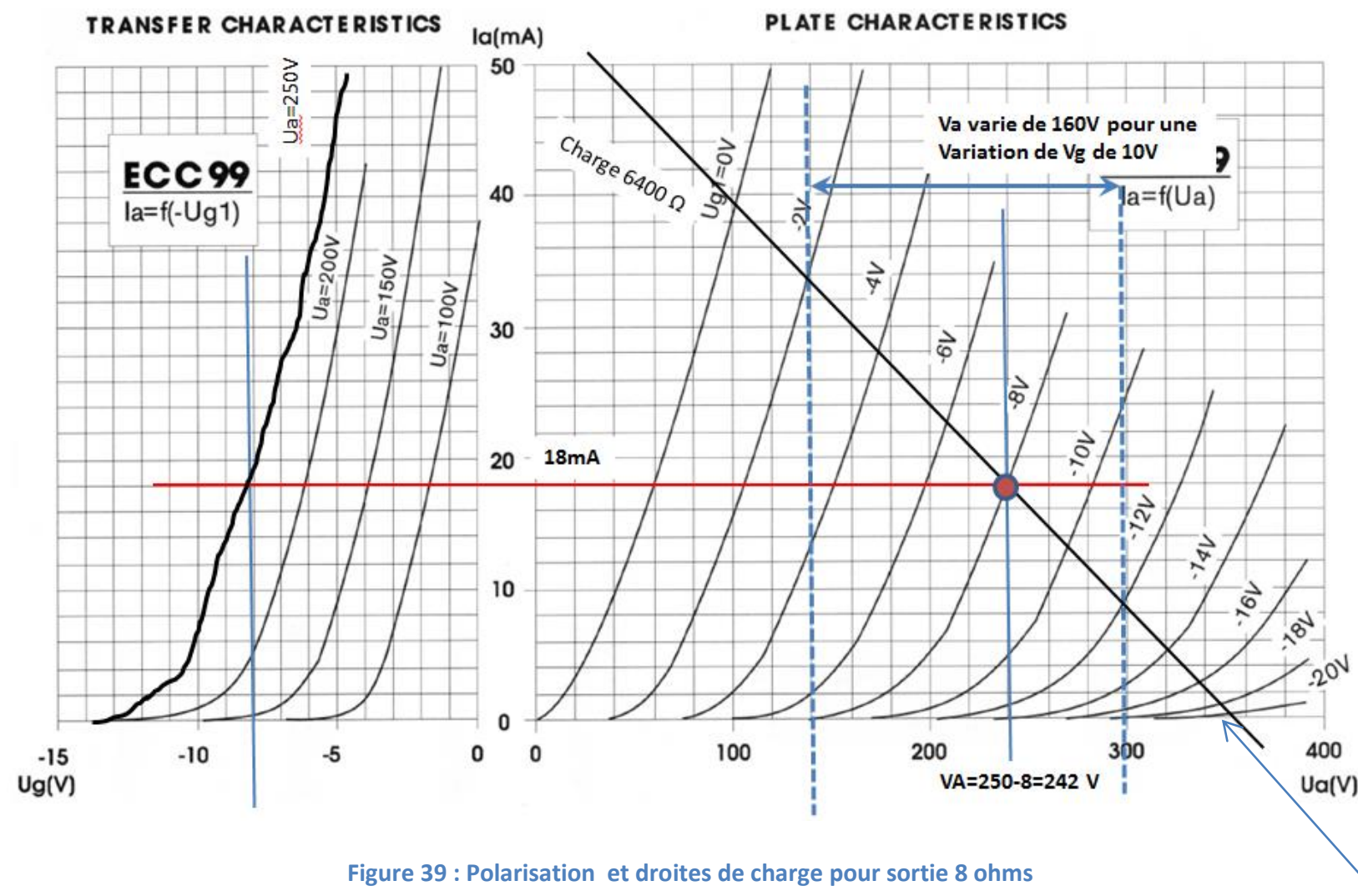

Ceci étant le gain ne varie pas considérablement puisqu'il reste approximativement de 16 par étage. On constate que l'excursion maximale positive (donc quand l'alternance d'entrée est négative) est de (350$242)=108 \mathrm{~V}$ au grand maximum (avec distorsion dans le haut) donc une valeur max d'entrée de $108 / 16=6,75 \mathrm{~V}$.

\subsection{Utilisation du simulateur pour la vérification des caractéristiques}

Comme pour le Fender, un schéma de départ (le préamplificateur) est fourni aux étudiants. Le modèle du transformateur de sortie leur est aussi fourni. Cela leur permet rapidement d'arriver au schéma de simulation de l'ensemble (Figure 43):

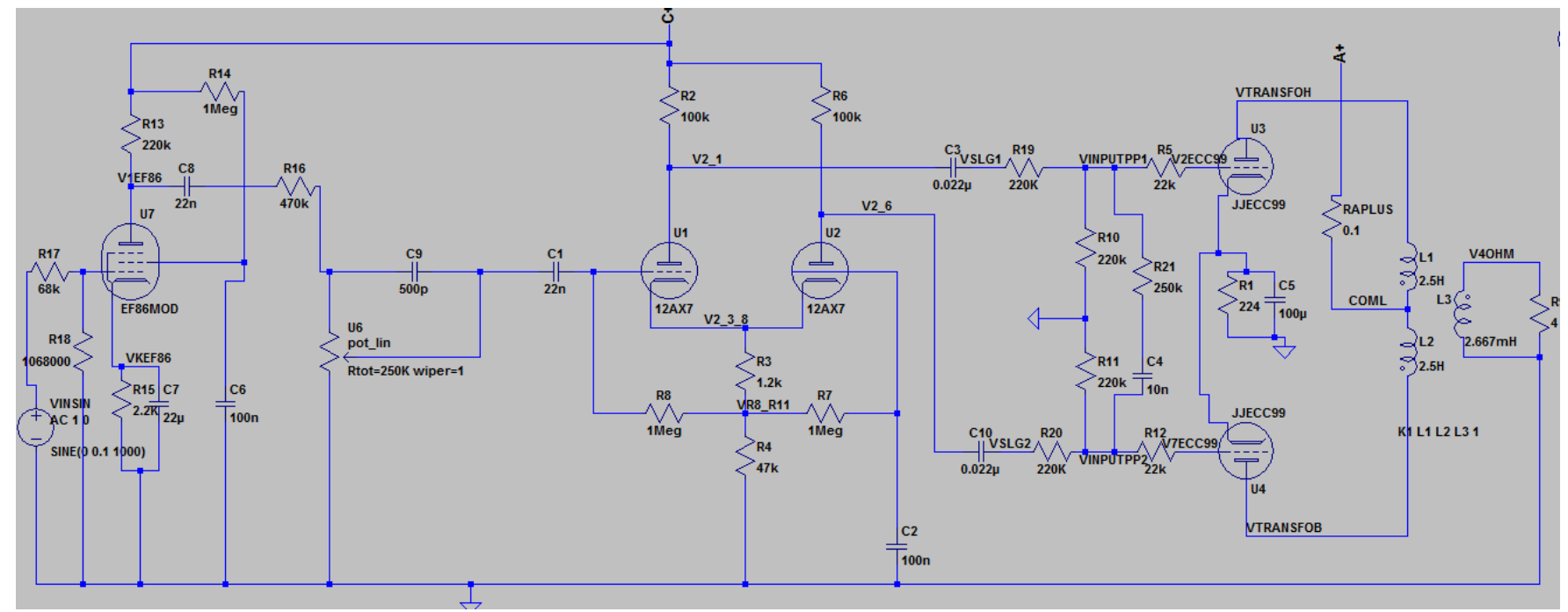

Figure 40 : Schéma de simulation VOX AC-30

Les résultats de simulation sont donnés sur les figures suivantes : 


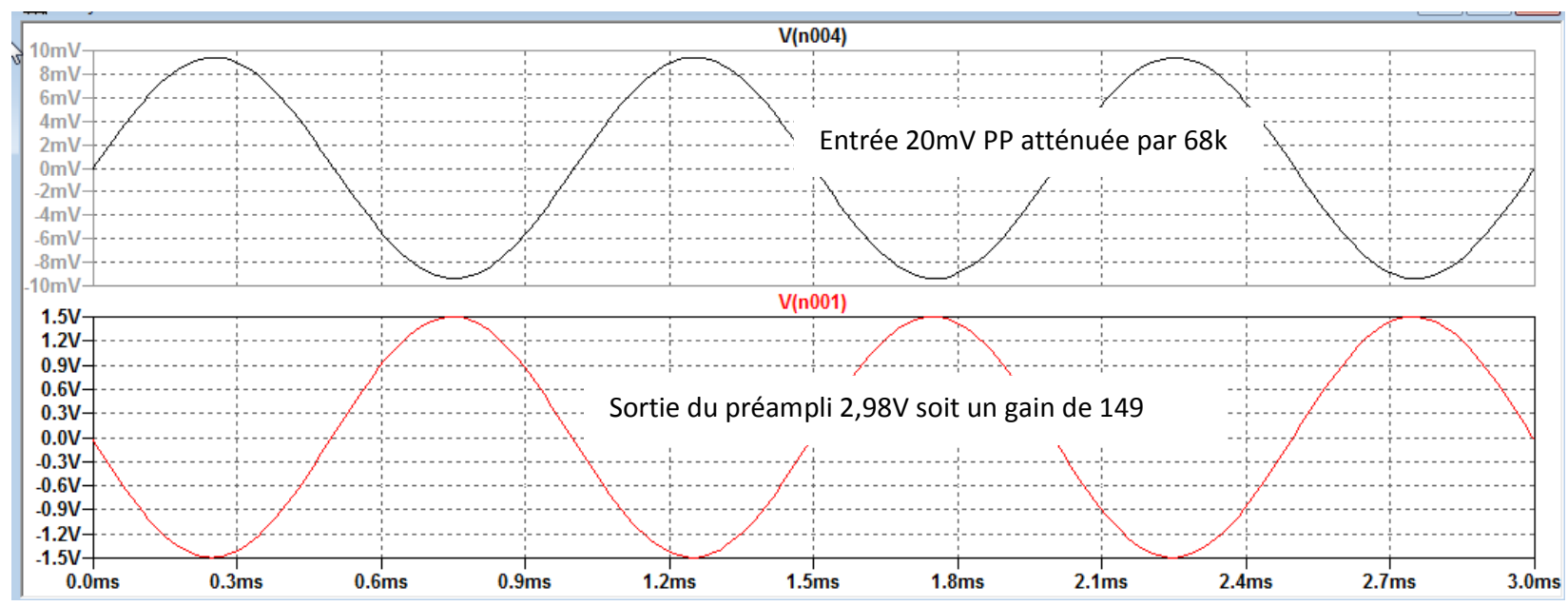

Figure 41 : Gain de l'étage d'amplification (entrée)

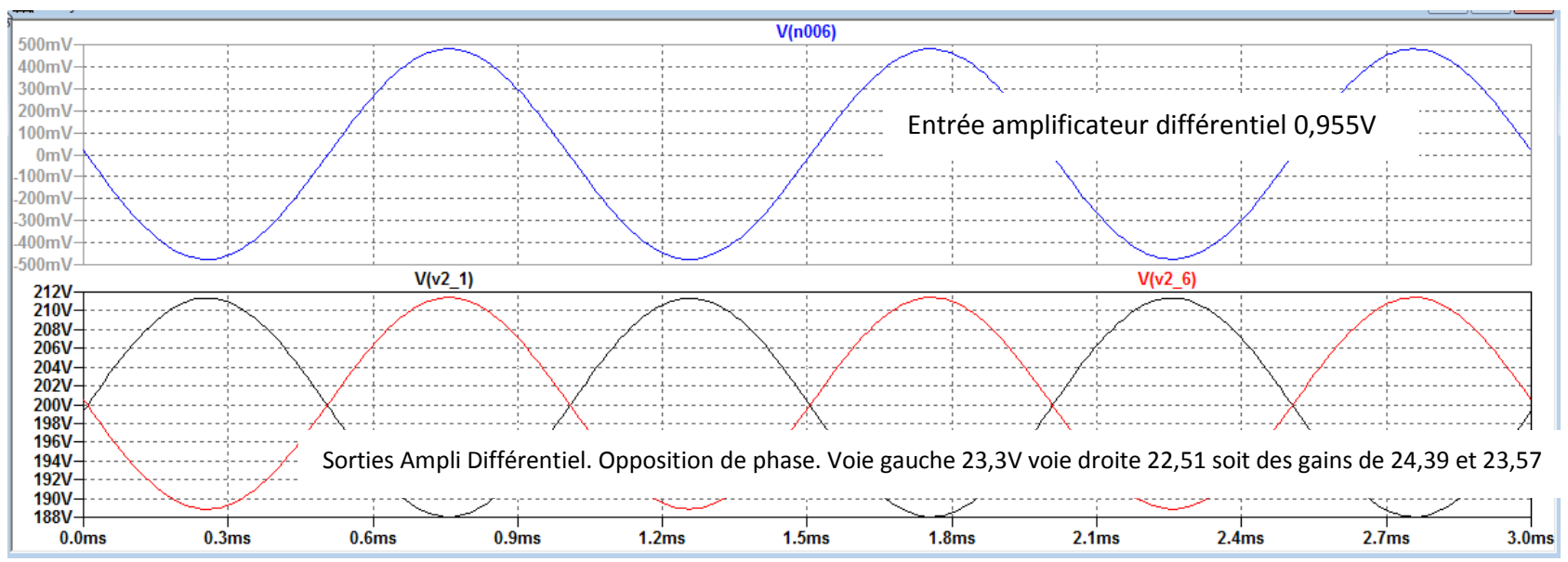

Figure 42 : Gain de l'amplificateur différentiel

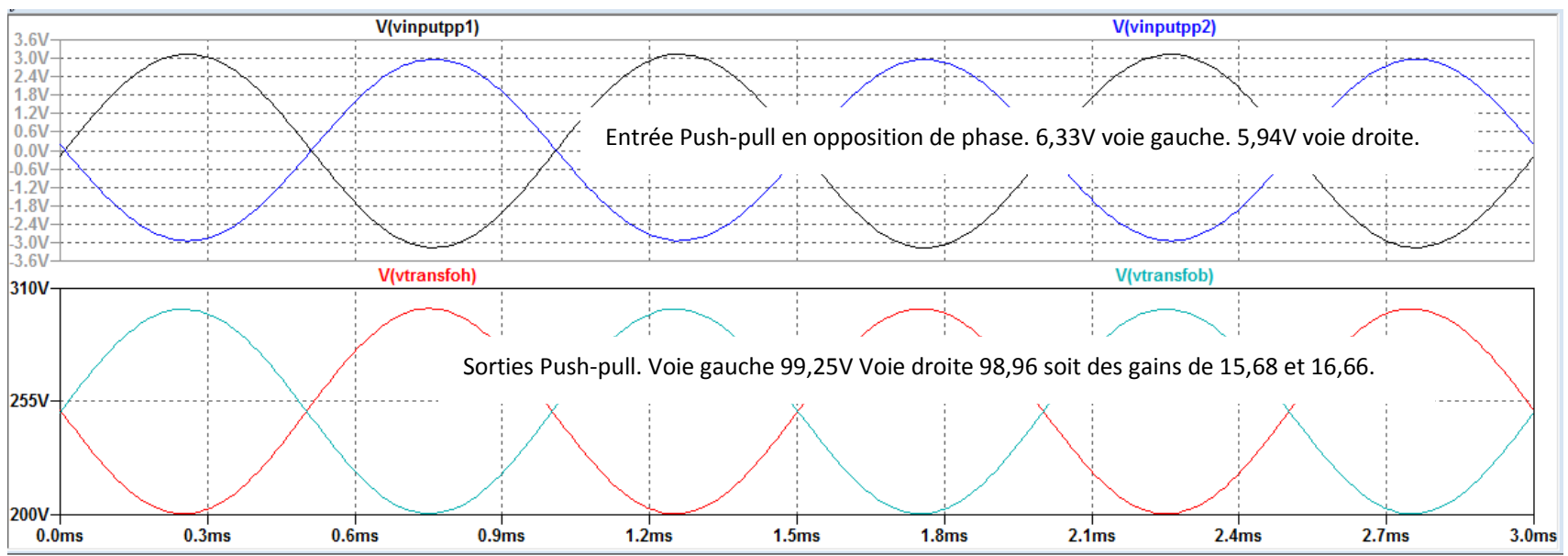

Figure 43 : Gain du Push-Pull de sortie 


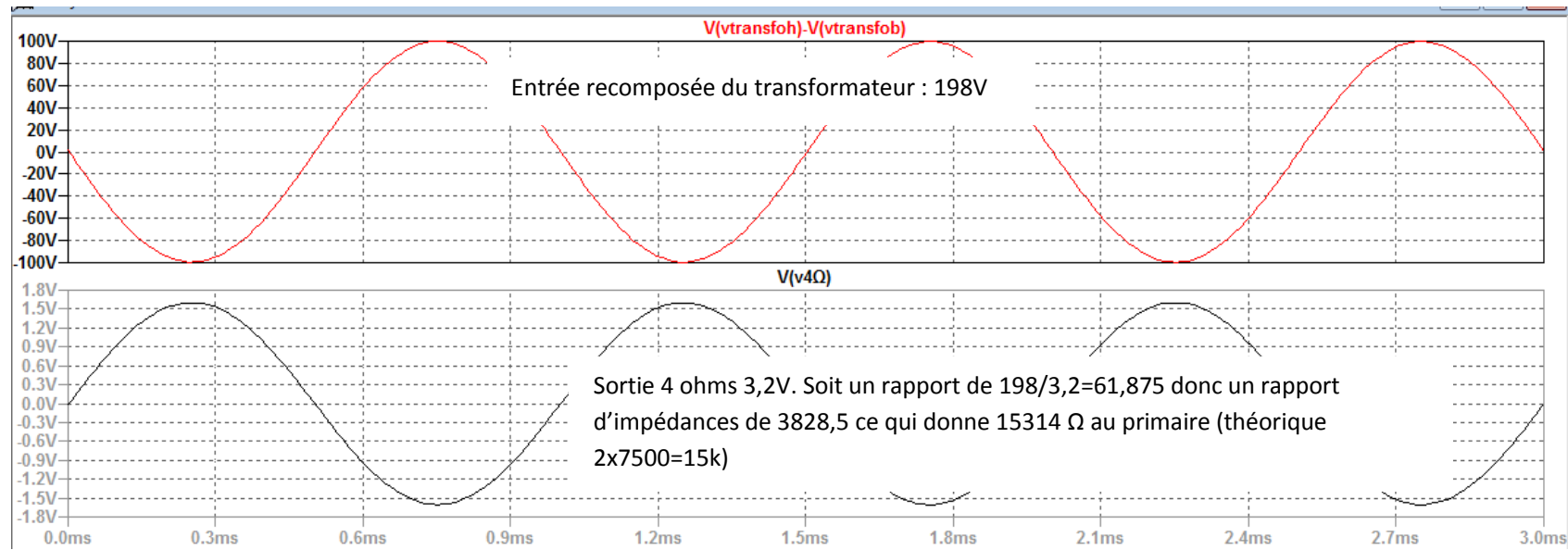

Figure 44 : Entrée du transformateur et sortie sur charge 4 ohms

\subsection{Montage de l'amplificateur}

Le montage a concerné 3 groupes d'étudiants par binôme. Un groupe pour l'alimentation, un groupe pour la « Turret board » et un groupe pour le châssis. Le résultat final est donné sur les figures 48 et 49 :

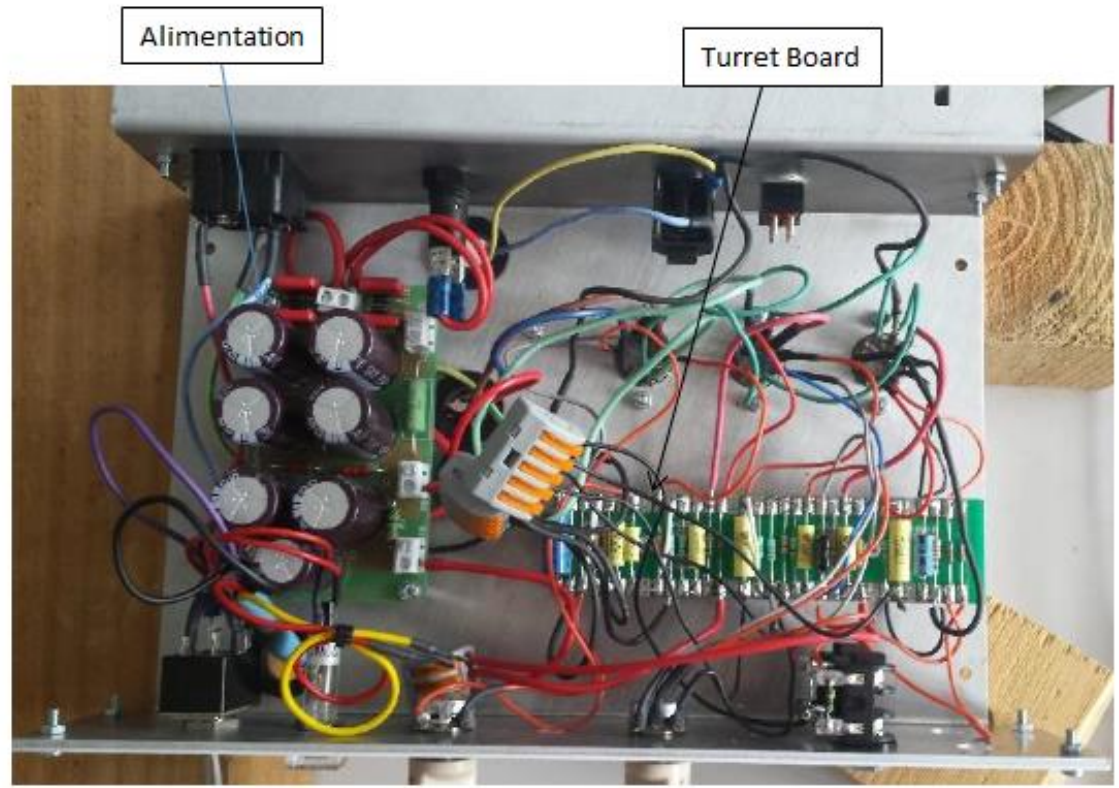

Figure 45 : Vue du câblage de l'Amplificateur

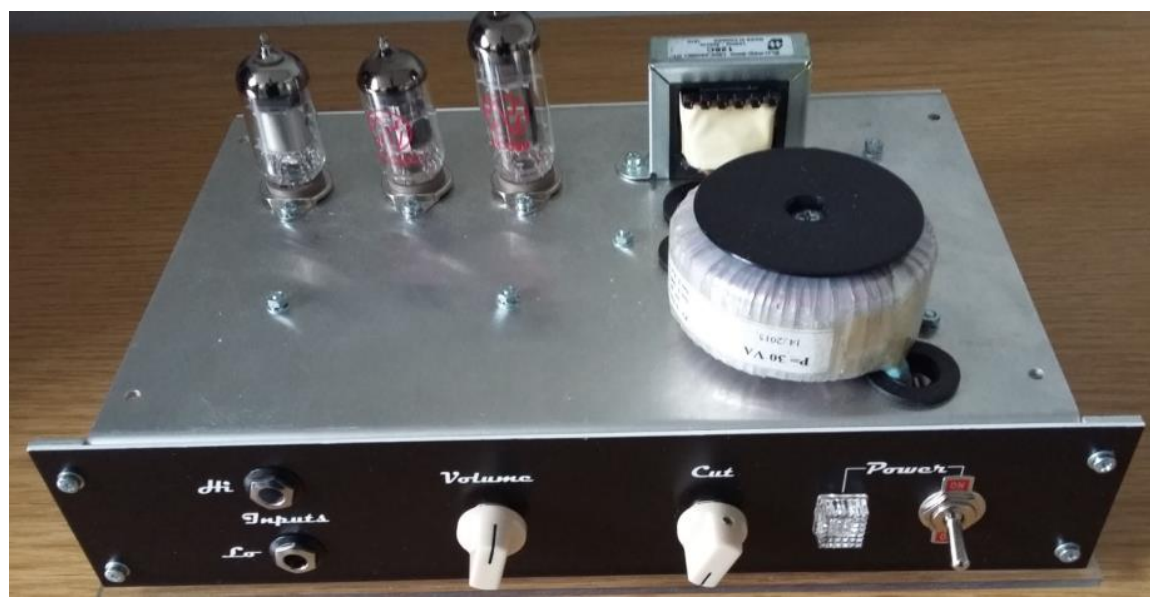

Figure 46 : L'amplificateur une fois terminé 


\subsection{Mesures et test}

Pour effectuer les mesures sans faire de bruit, l'amplificateur a été chargé par une résistance de puissance de 4 ohms. Les figures ci-dessous montrent qu'il y a peu d'écart entre les résultats théoriques et les relevés effectués.

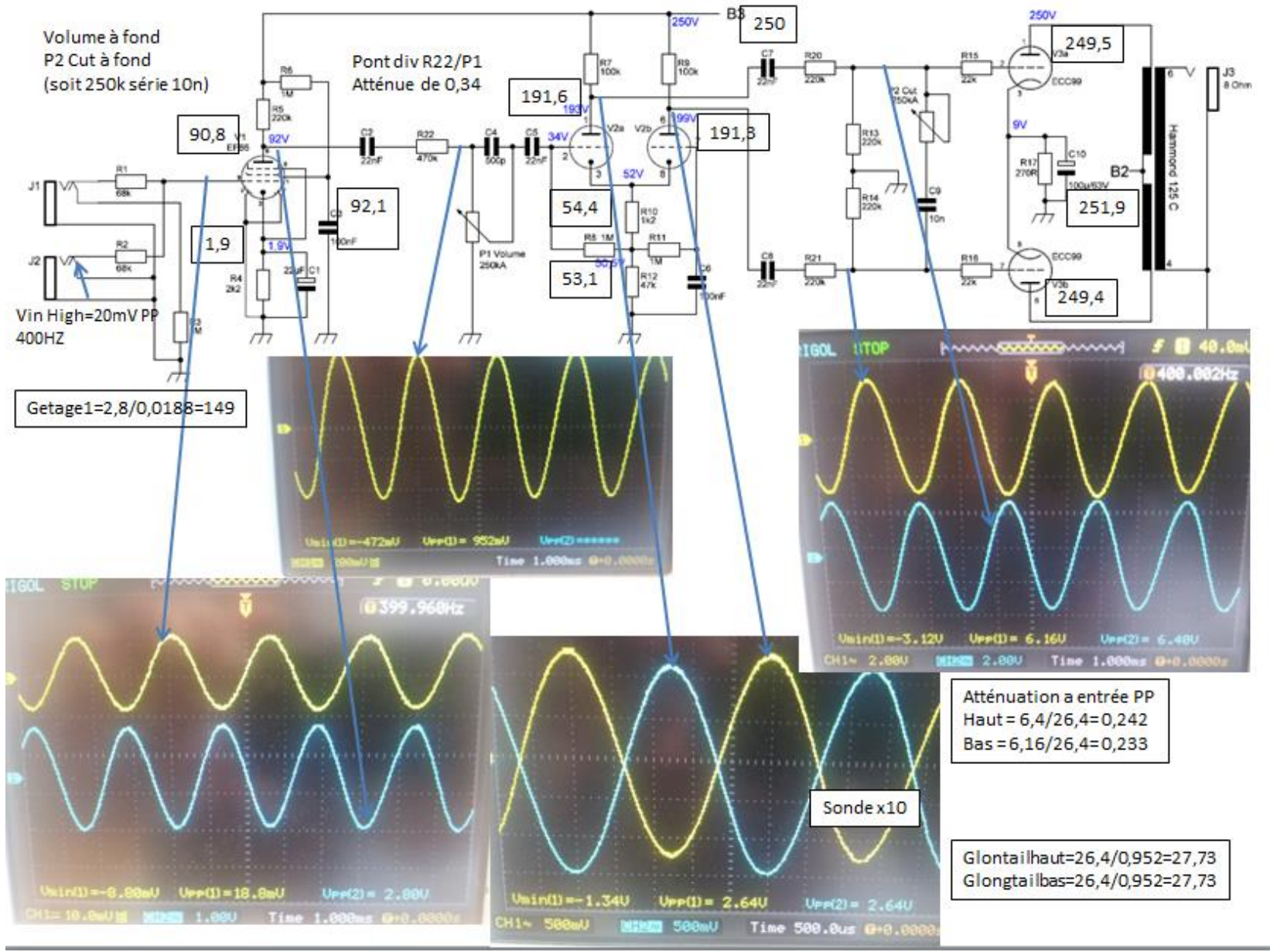

Figure 47 : Mesures entre l'entrée jusqu'à la sortie de l'étage différentielle 


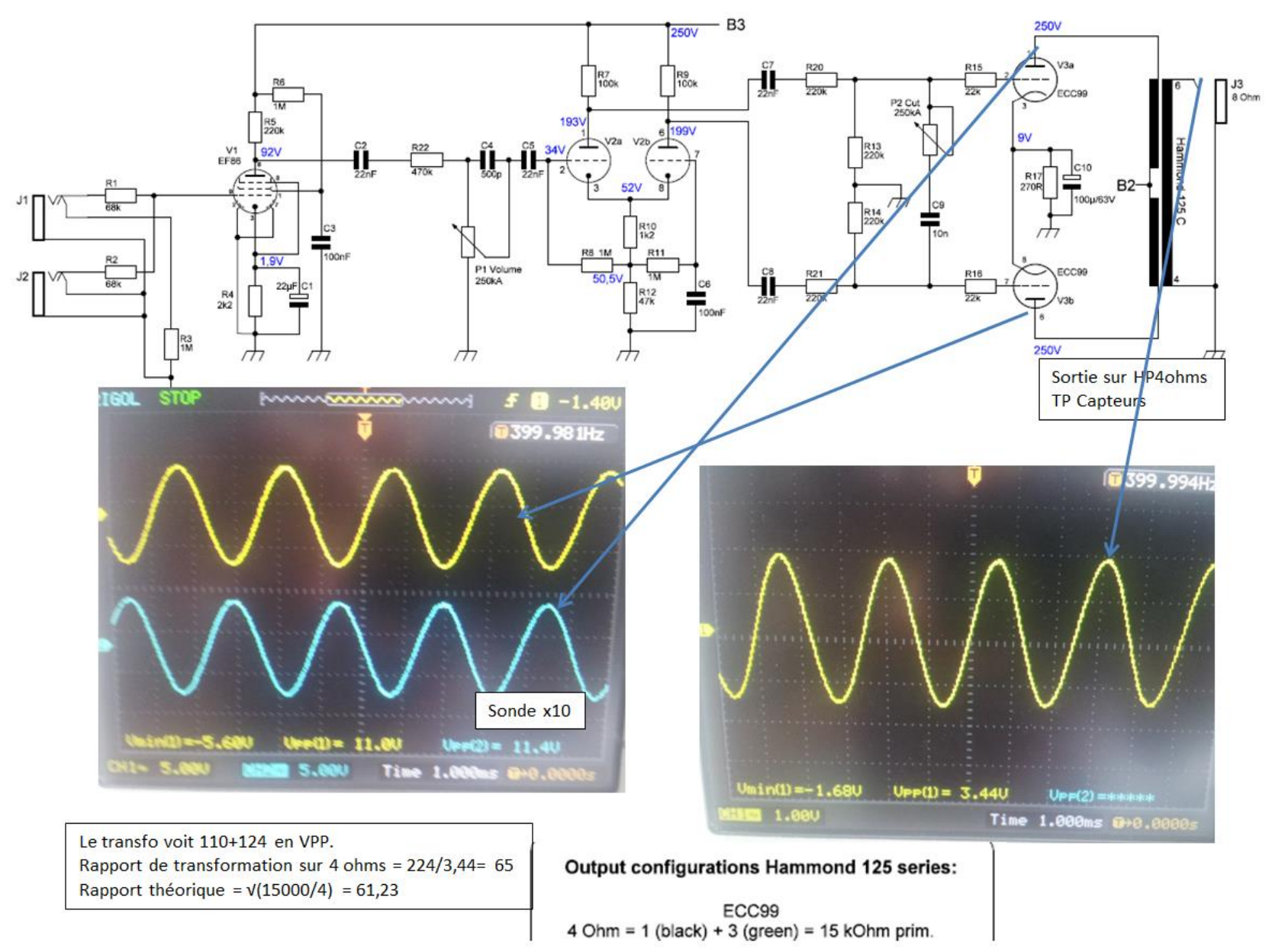

Figure 48 : Mesures et vérification du rapport de transformation et du rapport d'impédance

Le circuit de tonalité de base du VOX AC30 a été brièvement étudié par les étudiants, il s'agit en fait d'un filtrage passe-bas qui dépend de la valeur du potentiomètre de tonalité. La figure ci-dessous donne le relevé pour 3 positions du potentiomètre de « cut ».

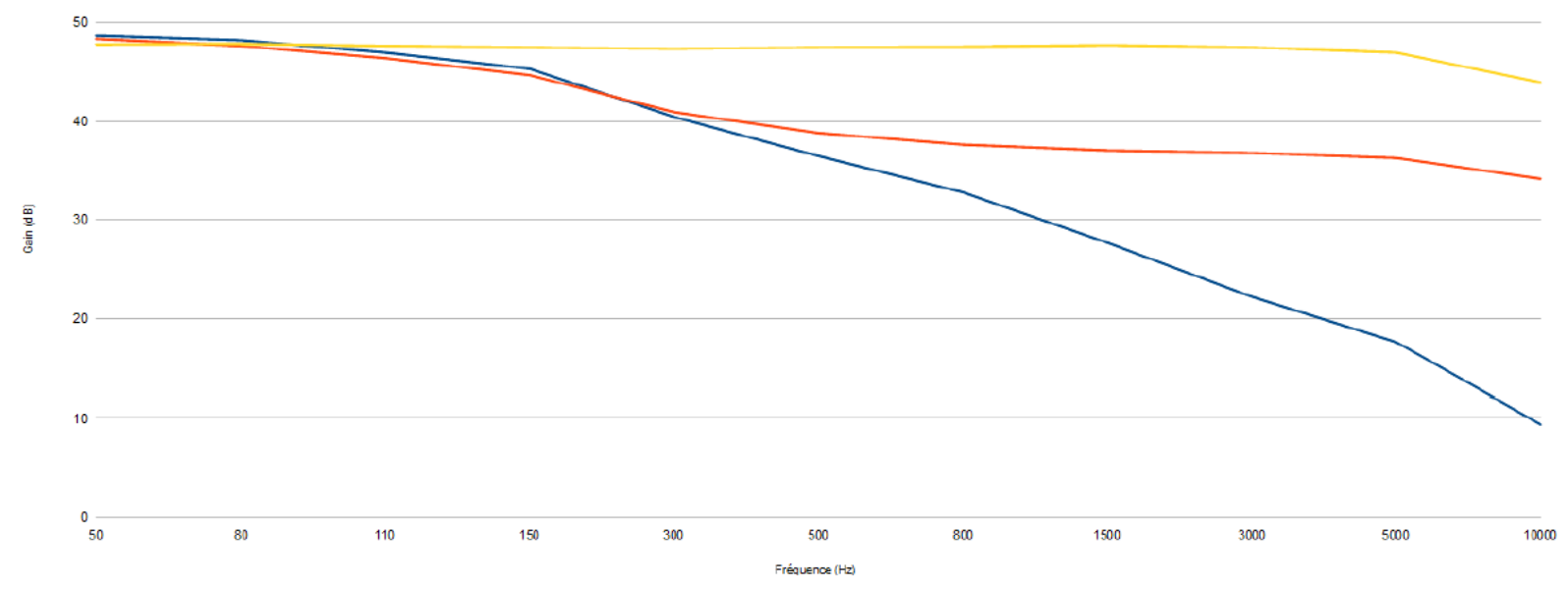

Figure 49 : Effet du potentiomètre de "cut" 


\subsection{Evolution du montage et mise en boitier}

Les étudiants ont souhaité ajouter un circuit de tonalité un peu plus évolué. Pour les aider dans leurs recherches et dans le choix du circuit le programme «Tone-Stack Calculator »[9] a été installé. Cela leur a permis de fixer leur choix sur un correcteur de son de type Fender/Marshall/Vox à 3 potentiomètres (Bass Middle Treble) [10] [11] . Le responsable du projet s'est chargé de vérifier l'adéquation du filtre proposé au montage de l'amplificateur et a proposé les modifications nécessaires dues à l'atténuation apportée par le circuit de tonalité.

Le fonctionnement du filtre fait apparaitre une atténuation d'environ $14 \mathrm{~dB}$ pour la courbe plate (Bass à 0 Treble à 0 et Middle à 1), voir figure ci-dessous.

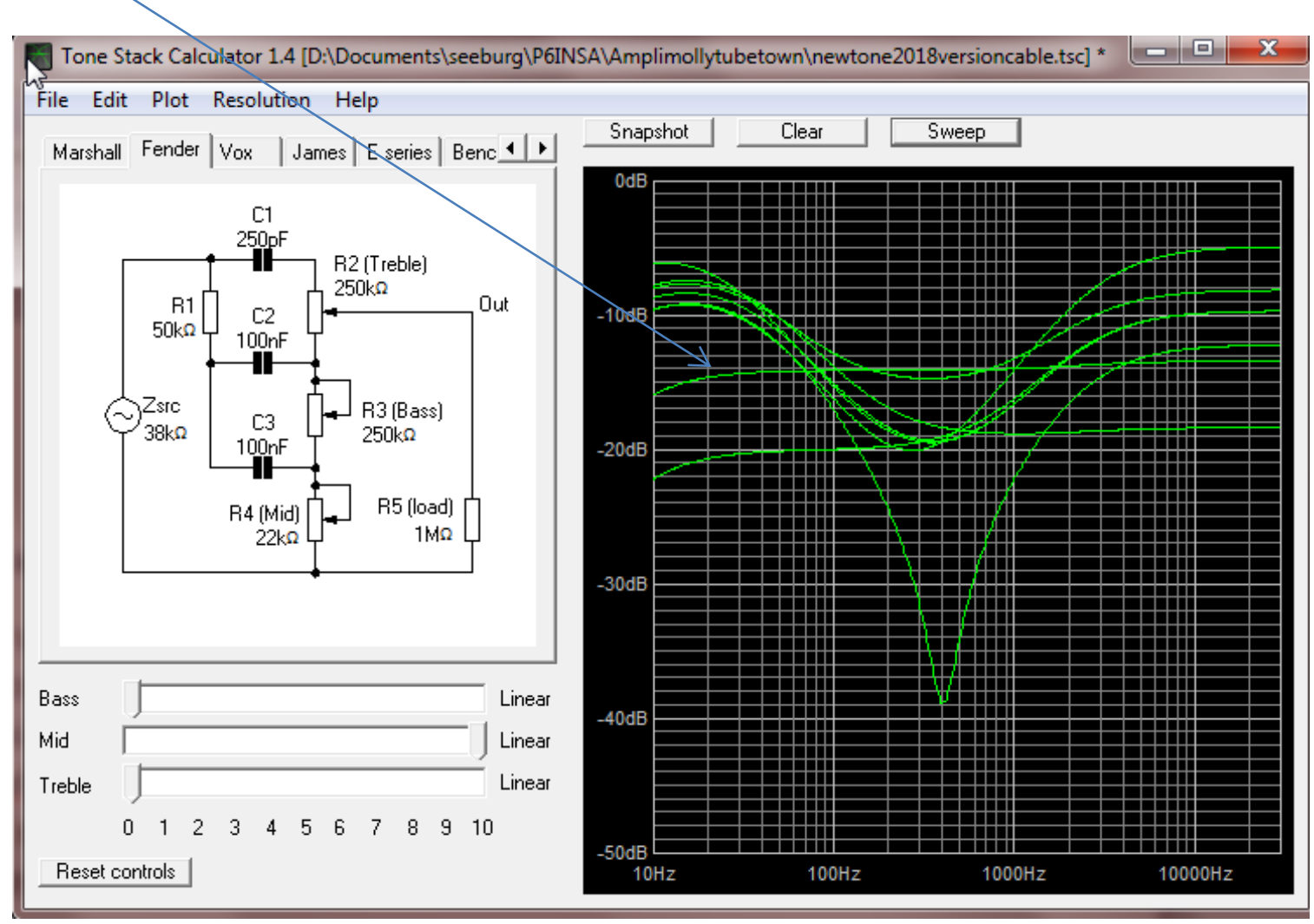

Figure 50 : Effet du correcteur de tonalité à 3 boutons

Les modifications apportées ont été les suivantes :

- Insertion du circuit de tonalité entre les condensateurs C4 et C5 (Les références des composants sont celles de la Figure 32).

- Changement de R22 de $470 \mathrm{k} \Omega$ à $10 \mathrm{k} \Omega$ pour diminuer l'atténuation en sortie du préampli

- Suppression du potentiomètre de «cut»

- Remplacement de R13 et R14 par des résistances de $1 \mathrm{M} \Omega$ pour supprimer l'atténuation en sortie de l'amplificateur différentiel

La Figure 54 montre les effets du circuit de tonalité sur la courbe de réponse. 

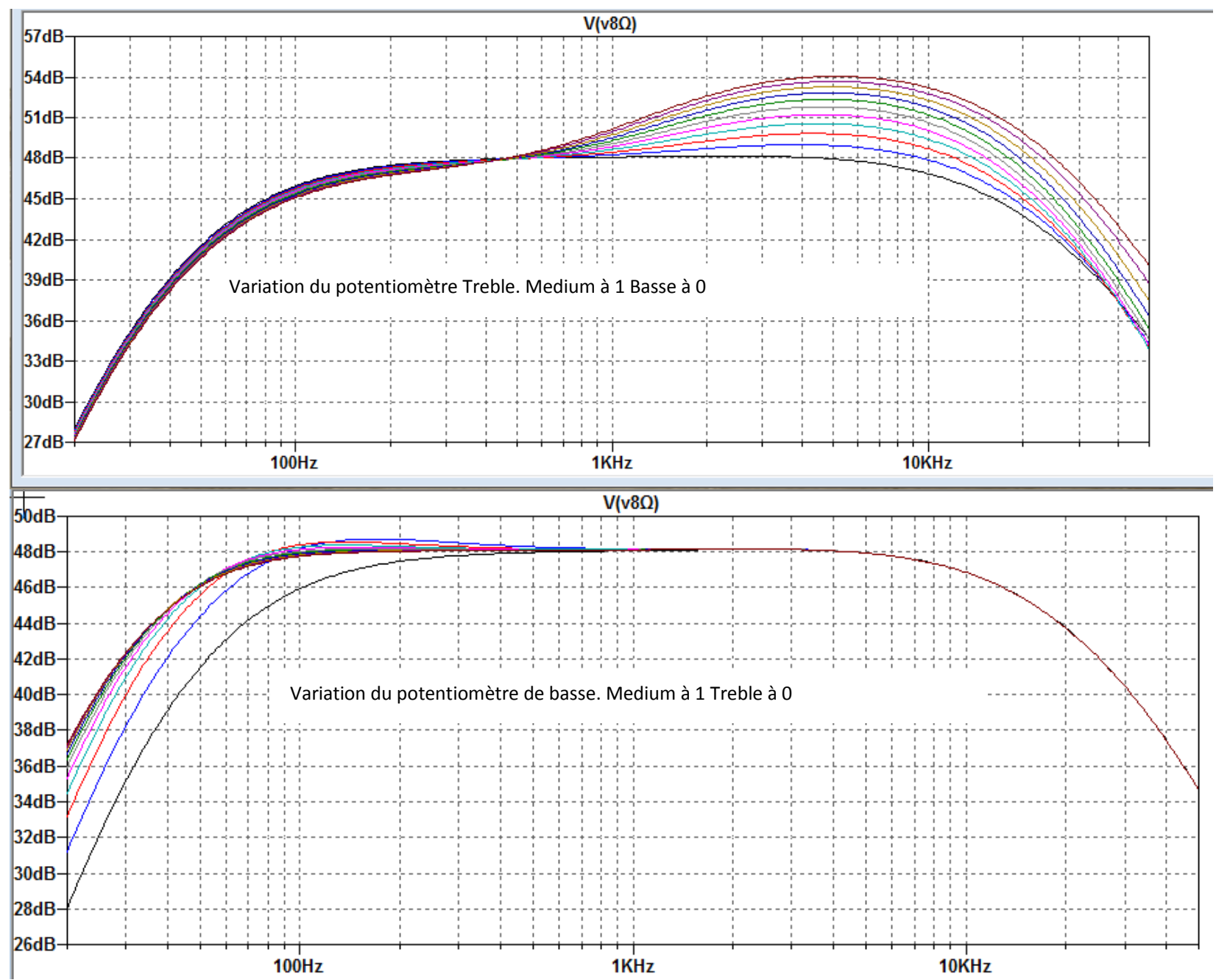

Figure 51 : Effets en simulation du correcteur de tonalité choisi (le montage initial avait un gain de 48dB)

Cela donne la nouvelle structure de l'amplificateur (Figure 55)

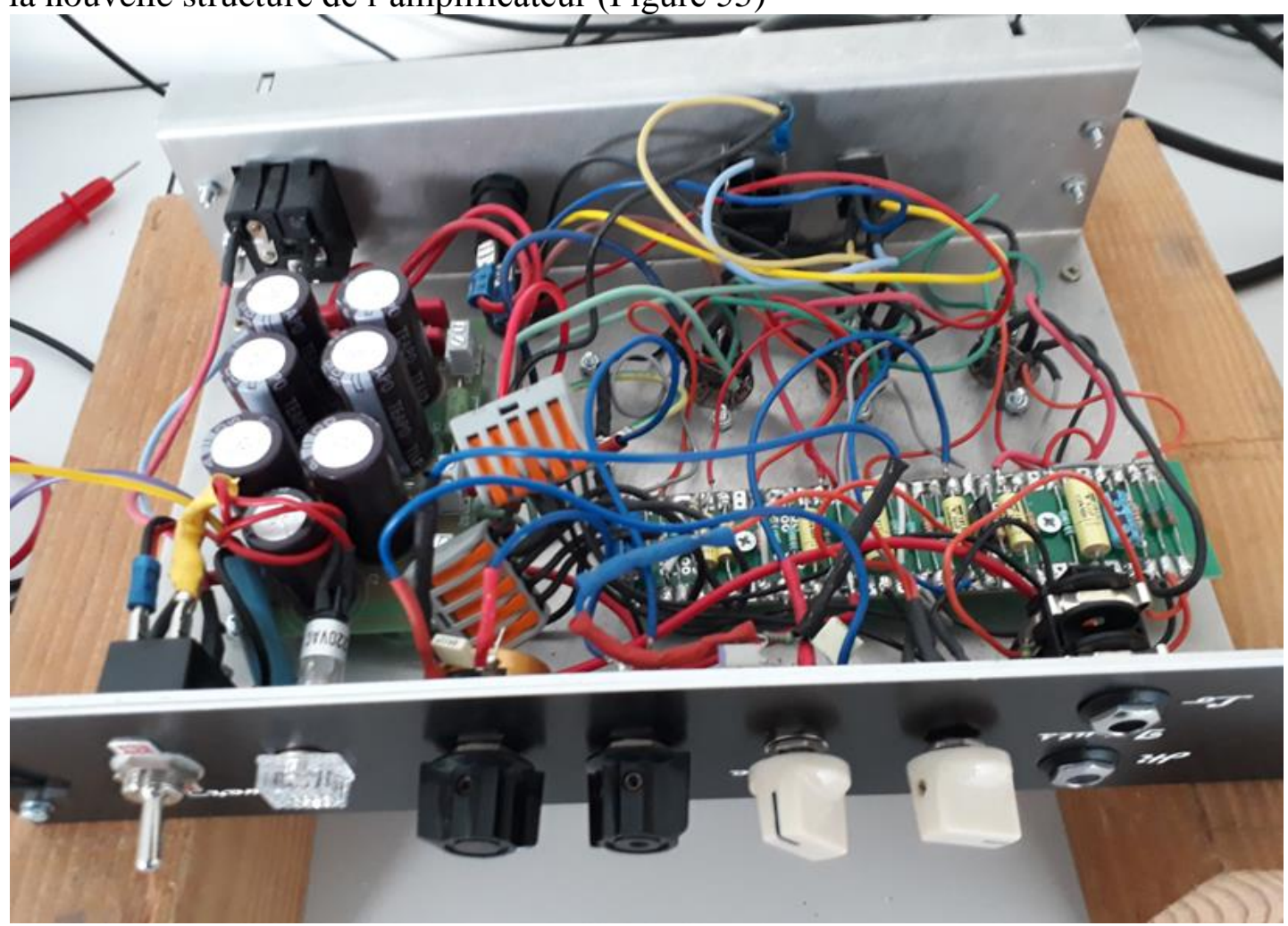

Figure 52 : Version AC-30 avec circuit de tonalité (Bass/Middle/Treble) 


\section{Conclusion}

Les remarques des étudiants donnent un bon aperçu de l'utilité de ce genre de projet imbriquant histoire de la musique, électronique, simulation, réalisation et tests.

Mae : «J'ai trouvé ce projet très intéressant. C'était passionnant de découvrir le fonctionnement d'un amplificateur à lampes et d'étudier ses fonctionnalités. C'est incroyable de voir le résultat lorsque l'on joue de la guitare. De plus, le projet était enrichissant, il m'a permis de mener un travail en groupe et de réaliser des mesures pour ensuite les analyser. »

Nhi : «Ce projet est une expérience inoubliable pour moi. Depuis très longtemps, je suis très intéressée par l'amplificateur à lampes. Grâce à ce projet, je peux mieux comprendre et bien découvrir ce type spécifique d'amplificateur. D'un autre côté, ça m'aide beaucoup à travailler en groupe avec des nouvelles personnes. »

Florent : «Ce projet m'a permis d'améliorer mon esprit d'équipe, ce qui est très important dans mon futur métier d'ingénieur où je serai forcément amené à travailler en groupe. De plus, ce projet m'a permis de découvrir le logiciel LTSPICE qui nous a été très utile pour la simulation du Fender. Enfin, j'ai pu mieux comprendre le fonctionnement de l'amplificateur à lampes ainsi que du circuit de tonalité. »

Corentin : «Ce projet m'a beaucoup apporté en terme de travail d'équipe : il m'a fait prendre conscience que la mise en commun et la séparation des tâches sont essentielles pour mener un projet à bien. Il m'a également permis de concevoir une modification sur l'ampli par nos propres moyens en faisant des recherches puis des simulations puis des tests réels, ce qui s'approche de la démarche d'un ingénieur. »

Vincent : " En tant que redoublant, c'est mon deuxième projet physique et je dois dire que j'ai aussi appris énormément de choses. Ça a été super agréable de travailler dans ce groupe car il y avait une très bonne ambiance de travail (partage des tâches, etc.). J'ai pu acquérir de nombreuses connaissances sur les amplificateurs à lampes et le logiciel LTSPICE et j'ai bien aimé l'instant «bricolage » lors de la construction du second circuit de tonalité. »

Le lien donné en référence [12] permet d'avoir une idée du son obtenu avec les amplificateurs.

\section{Remerciements}

L'auteur tient à remercier l'environnement technique pour l'électronique du département STPI : Pascal Williams, Hélène Rade, Michael Jolly ainsi que tous les étudiants qui ont eu à cœur de mener à bien la réalisation de ces projets.

\section{Références}

[1] https://www.fender.com/articles/gear/indispensable-undeniable-the-legacy-of-the-champ

[2] https://valvetubeguitaramps.com/vox-ac30-which-were-the-best-models/

[3] http://www.r-type.org/pdfs/12ax7.pdf

[4] https://www.jj-electronic.com/en/6v6s

[5] http://www.hammondmfg.com/pdf/5C_125SE.pdf

[6] Merlin Blencowe, Designing Valve Preamps for Guitar and Bass, Second Edition, 2012

[7] EF86 Pentode Data Sheet, http://www.r-type.org/exhib/aaq0204.htm

[8] ECC99 Double Triode data sheet, https://www.drtube.nl/datasheets/ecc99-ji2003.pdf

[9] Tone-Stack Calculator http://www.duncanamps.com/tsc/

[10] Fender Bassman 5F6-A Schematics with 3 Knobs Tone control. http://ampwares.com/schematics/bassman 5f6a.pdf

[11] Marshall JTM45 Basic Schematics with 3 knobs tone control. 
https://drtube.com/schematics/marshall/jtm45tr.gif

[12] https://youtu.be/i9x11p2-mAw test des amplis (version Fender avec circuit de tonalité, VOX AC30 circuit de tonalité d'origine) avec une guitare Guild StarFire pour l'amplificateur Fender et une Fender Stratocaster pour l'amplificateur VOX 